\title{
SINGULAR POINTS OF ORDINARY LINEAR
}

\section{DIFFERENTIAL EQUATIONS*}

\author{
BY \\ GEORGE D. BIRKHOFF
}

Let

$$
\frac{d y_{i}}{d x}=\sum_{j=1}^{n} a_{i j}(x) y_{j} \quad(i=1,2, \cdots, n)
$$

be a system of $n$ ordinary linear differential equations of the first order in which we have for $|x|>R$

$$
a_{i j}(x)=a_{i j} x^{q}+a_{i j}^{(1)} x^{q-1}+\cdots+a_{i j}^{(q)}+a_{i j}^{(q+1)} \frac{1}{x}+\cdots \quad(i, j=1,2, \cdots, n),
$$

$q$ being an integer. We propose to investigate the nature of the solutions of (1) in the vicinity of $x=\infty$, which is taken to be a singular point of the given system of equations so that $q \geqq-1$. The integer $q+1$ is termed the rank of (1) at $x=\infty$, after Poincare. $\dagger$ It will be further assumed that the $n$ roots

of the characteristic equation

$$
\alpha_{1}, \alpha_{2}, \cdots, \alpha_{n}
$$

$$
\left|a_{i j}-\delta_{i j} \alpha\right|=0
$$

[where $\delta_{i j}=0(i \neq j)$, and $\left.\delta_{i i}=1\right]$ are distinct, and in the case $q=-1$ do not differ by integers. The writer hopes to discuss the general case later.

It is apparent that any proper linear transformation

$$
\bar{y}_{i}=\sum_{j=1}^{n} \lambda_{i j}(x) y_{j},
$$

in which the functions $\lambda_{i j}(x)$ are analytic or have a pole at $x=\infty$, will transform (1) into a system of equations $(\overline{1})$ with coefficients $\bar{a}_{i j}(x)$ of the same form (2), although $\bar{q}$ is not necessarily equal to $q$. Any such system of equations ( $\overline{1})$ will be said to be equivalent to (1) at $x=\infty$.

The first part of the paper deals with the determination of the simplest system of differential equations which is equivalent to (1) at $x=\infty$. In $\S 1$ an important Lemma on Analytic Functions is stated and proved. The applica-

\footnotetext{
* Presented to the Society (Chicago), April 17, 1908, under a different title.
}

† Acta Mathematica, vol. 8 (1886), p. 305. 
tion of this lemma in $\S 2$ shows that there always exists a canonical system of equations equivalent to (1) at $x=\infty$ having the form

$$
x \frac{d y_{i}}{d x}=\sum_{j=1}^{n} P_{i j}(x) y_{j} \quad(i=1,2, \cdots, n),
$$

in which $P_{i j}(x)$ are polynomials. In $\S 3$ it is shown that the rank of this canonical system of equations at $x=\infty$ can be taken to be $q$ so that the degree of the polynomials $P_{i j}(x)$ does not exceed $q+1$.

The significance of these results is best seen by considering the case of a regular singular point $q=-1$. The canonical system of equations equivalent to $(1)$ is then in simplest form $(\S 3)$

$$
x \frac{d Y_{1}}{d x}=\alpha_{1} Y_{1}, \quad x \frac{d Y_{2}}{d x}=\alpha_{2} Y_{2}, \cdots, x \frac{d Y_{n}}{d x}=\alpha_{n} Y_{n}
$$

and a fundamental system of solutions is

$$
\begin{aligned}
& Y_{1}^{(1)}=x^{\alpha_{1}}, \quad Y_{2}^{(1)}=0, \quad \cdots, \quad Y_{n}^{(1)}=0, \\
& Y_{1}^{(2)}=0, \quad Y_{2}^{(2)}=x^{\alpha_{2}}, \quad \cdots, \quad Y_{n}^{(2)}=0, \\
& Y_{1}^{(n)}=0, \quad Y_{2}^{(n)}=0, \quad \cdots, \quad Y_{n}^{(n)}=x^{a_{n}} .
\end{aligned}
$$

Now the solutions of (1) and the above system of equations are connected by a relation

$$
y_{i}=\sum_{j=1}^{n} \lambda_{i j}(x) Y_{j} \quad(i=1,2, \cdots, n),
$$

in which the $\lambda_{i j}(x)$ are analytic at $x=\infty$ (or have a pole, but this case is easily excluded). It follows that there exists a fundamental system of solutions of $(1), y_{1}^{(j)}, y_{2}^{(j)}, \cdots, y_{n}^{(j)}(j=1,2, \ldots, n)$, of the form

$$
y_{i}^{(j)}=\sum_{k=1}^{n} \lambda_{i k}(x) Y_{k}^{(j)}=\lambda_{i j}(x) x^{\alpha_{j}} \quad(i, j=1,2, \cdots, n) .
$$

This is the fundamental theorem for a regular singular point. The solutions of the canonical system of equations equivalent to (1) at $x=\infty$ play the same rôle in the case of an irregular singular point $(q \geqq 0)$ that they do in the case above considered.

The nature of the solutions of the canonical system of equations can be discussed by special and elegant methods when $q \geqq 0$. In the case $q=0$ it is known that the solutions of any system (1) with rational coefficients $a_{i j}(x)$ can be expressed by a Laplace integral

$$
y_{i}=\int e^{x z} \eta_{i}(z) d z \quad(i=1,2, \cdots, n),
$$


and that from this formula can be deduced the behavior of the solutions in the vicinity of $x=\infty$. This method of treatment is due to PoIncare,* and has been developed more fully by HoRN. $\dagger$ It is applicable in an extremely simple form to canonical systems of equations for which $q=0$. If $q>0$, generalized Laplace integrals of a simple character will be shown to be available. The integrals for $q \geqq 0$ are considered in $\S 4$.

These integrals are employed in $\S 5$ to find the asymptotic nature of the solutions of the canonical system of equations equivalent to (1) at $x=\infty$ in certain sectors of the $x$-plane. The asymptotic form of the solutions of $(1)$ in the same sectors follows as an immediate consequence. The powerful and general method of successive approximation which HoRN $\ddagger$ has employed in various cases will no doubt lead to the same results.

A Riemannian characterization of the solutions of the canonical system of equations in the vicinity of $x=\infty$ is given and extended in $\S 6$ to the solutions of (1). The number of characteristic constants in the solutions is found to be the same as the number of arbitrary constants of the canonical system of equations.

The characterization suggests the probable generalization of the Riemannian problem when the singular points of the system of differential equations are not regular. This formulation and a count of constants is given in $\S 7$.

\section{§1. Auxiliary lemma.}

In order to obtain the simplest possible system $(\overline{1})$ equivalent to (1), we can choose as we please the $n^{2}$ functions $\lambda_{i j}$ of the transformation (4) but with the restriction that they are analytic or have poles at $x=\infty$ and that the determinant $\left|\lambda_{i j}\right|$ is not identically zero. How can one choose the functions $\lambda_{i j}$ so as to free as far as possible the solutions of $(\overline{1})$ from singularities in the finite plane? The answer to this question, which will be fundamental in our study of (1), is derived from the following lemma.

Lemma on analytic FUnCtions. Let $\theta_{i j}(x)$ be a set of $n^{2}$ functions of $x$, single-valued and analytic for $|x|>R$ but not necessarily analytic at $x=\infty$, and also such that the determinant

$$
\left|\theta_{i j}(x)\right| \neq 0
$$

$(|x|>R)$.

Then it is possible to so choose $n^{2}$ multipliers $\lambda_{i j}(x)$, analytic at $x=\infty$, that

$$
\sum_{i=1}^{n} \lambda_{i j}(x) \theta_{j k}(x)=x^{-m} \xi_{i k}(x), \quad\left(\text { or } \sum_{j=1}^{n} \theta_{i j}(x) \lambda_{j k}(x)=x^{-m} \xi_{i k}(x)\right)
$$

$$
(i, k=1,2, \cdots, n) \text {, }
$$

* American Journal of Mathematics, vol. 7 (1885), pp. 203-258. A single linear differential equation of the $n$th order is treated in this and other papers referred to later.

† Mathematisohe Annalen, vol. 50 (1897), pp. 525-556.

†Crelle's Journal, vol. 133 (1907), pp. 19-67. 
where $m$ is a fixed positive integer or zero, and the $\xi_{i k}(x)$ are entire functions of $x$ for which the determinant $\left|\xi_{i k}(0)\right| \neq 0$.

Proof. The two alternative forms of the Lemma are clearly equivalent. We shall confine ourselves to the proof of the second of these, which is the easier to give in matrix notation.

In order to elucidate the lemma let us consider first the case $n=1$. We have to determine $\lambda_{11}$ so that

$$
\theta_{11} \lambda_{11}=x^{-m} \xi_{11} \text {. }
$$

Since $\theta_{11}(x) \neq 0$ for $|x|>R$, the function $\log \theta_{11}$ is expansible in a Laurent series for $|x|>R$ except for a term $\alpha \log x$,

Take then

$$
\log \theta_{11}=\sum_{v=-\infty}^{+\infty} r_{v} x^{v}+\alpha \log x .
$$

if $\alpha<0$, or

$$
\lambda_{11}=e^{-\sum_{v=-1}^{-\infty} r_{v} x^{v}}, \quad m=\alpha, \quad \xi_{11}=e^{\sum_{v=0}^{+\infty} r_{v} x^{v}},
$$

$$
\lambda_{11}=e^{-\sum_{\nu=-1}^{-\infty} r_{v} x v}, \quad m=0, \quad \xi_{11}=x^{a} e^{\sum_{v=0}^{+\infty} r_{v} x v},
$$

if $\alpha \geqq 0$, and we have the desired solution. This explicit treatment is not possible if $n>1$, in which case we shall have to construct a solution by an infinite process.

As it is convenient to use matrix notation, let $\Theta$ denote the matrix $\left\|\theta_{i j}\right\|$ and $\Theta^{-1}$ its inverse matrix $\left\|\theta_{i j}^{-1}\right\|$ which is of the same form, since $\left|\theta_{i j}\right| \neq 0$ for $|x|>R$. Also we shall write $\Lambda=\left\|\lambda_{i j}\right\|$.

Furthermore, if $\phi$ is single-valued and analytic in the vicinity of $x=\infty$, so that it is expansible in a Laurent series,

we write

$$
\phi=\sum_{v=-\infty}^{+\infty} \alpha_{v} x^{v}
$$

$$
P(\phi)=\sum_{v=0}^{+\infty} \alpha_{v} x^{v}, \quad N(\phi)=\sum_{v=-1}^{-\infty} \alpha_{v} x^{v}, \quad N_{m}(\phi)=\sum_{v=-m}^{-\infty} \alpha_{v} x^{v} .
$$

Here $m$ is some positive integer whose value will be specified later. We have the obvious relations

$$
\begin{gathered}
\phi=P(\phi)+N(\phi)=P(\phi)+\alpha_{-1} x^{-1}+\cdots+\alpha_{-m+1} x^{-m+1}+N_{m}(\phi), \\
P[N(\phi)]=P\left[N_{m}(\phi)\right]=N[P(\phi)]=N_{m}[P(\phi)]=0 .
\end{gathered}
$$

More generally, if we have a matrix $\Phi \equiv\left\|\phi_{i j}\right\|$, we shall use the notation

$$
P(\Phi) \equiv\left\|P\left(\phi_{i j}\right)\right\|, \quad N(\Phi) \equiv\left\|N\left(\phi_{i j}\right)\right\|, \quad N_{m}(\Phi) \equiv\left\|N_{m}\left(\phi_{i j}\right)\right\| .
$$

Trans. Am. Math. Soc. 29 
Let now $T=\left\|t_{i j}\right\|$ be any matrix of constants of determinant not zero. Consider the matrix equations

$$
\Gamma=P\left(\Theta^{-1} \cdot \Psi\right) \quad \Psi=T x^{-m}+N_{m}(\Theta \cdot \Gamma) .
$$

If a solution of these equations $\Gamma=\left\|\gamma_{i j}\right\|, \Psi=\left\|\psi_{i j}\right\|$ exists, we will have at once the desired matrix

$$
\Lambda=N\left(\Theta^{-1} \cdot \Psi\right) \text {. }
$$

To verify this statement we notice first that the functions $\lambda_{i j}$ are analytic at $x=\infty$ by (6), since they are convergent series of negative powers of $x$. Moreover from the first equation of (5) we obtain

$$
\Gamma+N\left(\Theta^{-1} \cdot \Psi\right)=\Theta^{-1} \cdot \Psi .
$$

From this equation (7) we derive

$$
\Theta \cdot \Gamma+\Theta\left[N\left(\Theta^{-1} \cdot \Psi\right)\right]=\Psi
$$

on multiplying by the matrix $\Theta$. Furthermore, by the second equation of (5), we obtain also

$$
\Psi=T x^{-m}+\Theta \cdot \Gamma-P(\Theta \cdot \Gamma)-R,
$$

in which the elements of $R$ are polynomial of degree $m-1$ at most in $1 / x$. If we add (8) and (9), we find by means of $(6)$

$$
\Theta \cdot \Lambda=T x^{-m}-P(\Theta \cdot \Gamma)-R .
$$

The right hand member forms a matrix $\left\|x^{-m} \xi_{i j}(x)\right\|$ in which the $\xi_{i j}(x)$ are entire functions of $x$ with the desired property

$$
\left|\xi_{i j}(0)\right|=\left|t_{i j}\right| \neq 0 \text {. }
$$

We have thus proved our statement.

It remains to construct a solution of equations (5) for some $m$. This we do by a method of successive approximation.

Write

$$
\begin{aligned}
& \Psi_{0}=T x^{-m}, \\
& \Gamma_{0}=P\left(\Theta^{-1} \cdot \Psi_{0}\right), \\
& \Psi_{1}=T x^{-m}+N_{m}\left(\Theta \cdot \Gamma_{0}\right), \\
& \Gamma_{1}=P\left(\Theta^{-1} \cdot \Psi_{1}\right), \\
& \Psi_{2}=T x^{-m}+N_{m}\left(\Theta \cdot \Gamma_{1}\right),
\end{aligned}
$$

If the sequence of matrices $\Psi_{p}, \Gamma_{p}$ tend to limit matrices, it will not be difficult to prove that these limits form the desired solutions. 
Define the difference matrices

$$
\begin{array}{ll}
\bar{\Gamma}_{p}=\Gamma_{p}-\Gamma_{p-1}, & \bar{\Gamma}_{0}=\Gamma_{0}, \\
\bar{\Psi}_{p}=\Psi_{p}-\Psi_{p-1}, & \bar{\Psi}_{0}=\psi_{0} .
\end{array}
$$

These matrices for $p>0$ satisfy the equations

$$
\bar{\Psi}_{p}=N_{m}\left(\Theta \cdot \bar{\Gamma}_{p-1}\right), \quad \Gamma_{p}=P\left(\Theta^{-1} \cdot \bar{\Psi}_{p}\right) .
$$

It follows from the first of these equations that $\bar{\Psi}_{p}$ contains only negative powers of $x$. Hence the positive powers which come from the multiplication of $\Theta^{-1}$ and $\bar{\Psi}_{p}$ in the second of these equations arise from the positive powers in the elements of $\Theta^{-1}$ and from the negative in $\bar{\Psi}_{p}$ so that

Likewise we find

$$
P\left(\Theta^{-1} \cdot \bar{\Psi}_{p}\right)=P\left[P\left(\Theta^{-1}\right) \cdot \bar{\Psi}_{p}\right] .
$$

since the elements of $\bar{\Gamma}_{p_{-1}}$ contain only positive powers of $x$ and a constant term. Thus (11) can be written

$$
\bar{\Psi}_{p}=N_{m}\left[N_{m}(\Theta) \cdot \bar{\Gamma}_{p-1}\right], \quad \bar{\Gamma}_{p}=P\left[P\left(\Theta^{-1}\right) \cdot \bar{\Psi}_{p}\right] .
$$

In this form we shall prove the convergence of

$$
\Gamma=\bar{\Gamma}_{0}+\bar{\Gamma}_{1}+\cdots, \quad \Psi=\bar{\Psi}_{0}+\bar{\Psi}_{1}+\cdots
$$

for large enough $m$.

Choose $r_{0}, R_{0}$ so that

$$
r_{0}>R_{0}>R
$$

then we can choose $M$ so large that*

$$
P\left(\theta_{i j}^{-1}\right) \ll \frac{M}{1-\frac{x}{r_{0}}}=M\left(1+\frac{x}{r_{0}}+\frac{x^{2}}{r_{0}^{2}}+\cdots\right)
$$

since $P\left(\theta_{i j}^{-1}\right)$ is an entire function of $x$. Moreover since $N\left(\theta_{i j}\right)$ is analytic for $|x|>R$, the series for $N\left(\theta_{i j}\right)$,

$$
\frac{\theta_{i j}^{(1)}}{x}+\frac{\theta_{i j}^{(2)}}{x^{2}}+\cdots
$$

* The notation, $\alpha \ll \beta$, means that the coefficients of the various powers of $x$ in the power series $\alpha$ do not exceed the corresponding terms of $\beta$ in absolute value. I shall extend this notation of PoINCARÉ to matrices $A$ and $B$ of which corresponding terms $\alpha_{i j}$ and $\beta_{i j}$ are power series. Thus the notation $A \ll B$ implies that $\alpha_{i j} \ll \beta_{i j}$ for all values of $i$ and $j$. 
is convergent for $|x|>R$, so that

$$
\lim _{p=\infty} \frac{\theta_{i j}^{(p)}}{R_{0}^{p}}=0 .
$$

Hence for large enough $m$ we have $\left|\theta_{i j}^{(p)}\right|<\epsilon R_{0}^{p}, p>m$, no matter how small $\epsilon$ be taken. Thus we can choose $m$ so large that for any small $\epsilon$

Take $m$ so large that

$$
N_{m}\left(\theta_{i j}\right) \ll \frac{\epsilon}{\frac{x}{R_{0}}-1}=\epsilon\left(\frac{R_{0}}{x}+\frac{R_{0}^{2}}{x^{2}}+\cdots\right) .
$$

$$
\frac{n^{2} \epsilon M r_{0} R_{0}}{\left(r_{0}-R_{0}\right)^{2}}<1
$$

It is to be noticed that both the comparison series used converge for $r_{0}>|x|>R_{0}$.

Now choose $L$ so large that

First by (16) and (18) we see that

$$
\bar{\Gamma}_{0}=\Gamma_{0} \ll\left\|\frac{L}{1-\frac{x}{r_{0}}}\right\|
$$

$$
\begin{aligned}
\bar{\Psi}_{1}= & N_{m}\left[N_{m}(\Theta) \cdot \Gamma_{0}\right] \\
& \ll\left\|N_{m}\left(\frac{n \epsilon L r_{0} R_{0}}{\left(x-R_{0}\right)\left(r_{0}-x\right)}\right)\right\|=\left\|N_{m}\left[\frac{n \epsilon L r_{0}}{\left(r_{0}-R_{0}\right)}\left(\frac{1}{x-R_{0}}+\frac{1}{r_{0}-x}\right)\right]\right\| \\
& \ll\left\|\frac{n \epsilon L r_{0} R_{0}}{\left.\| r_{0}-R_{0}\right)\left(x-R_{0}\right)}\right\|^{*}
\end{aligned}
$$

Next, by (15) and the above inequality, we see that

$$
\bar{\Gamma}_{1}=P\left[P\left(\Theta^{-1}\right) \cdot \bar{\Psi}_{1}\right] \ll\left\|P\left[\frac{n^{2} \epsilon M L r_{0}^{2} R_{0}}{\left(r_{0}-R_{0}\right)\left(r_{0}-x\right)\left(x-R_{0}\right)}\right]\right\| \ll\left\|\frac{n^{2} \epsilon M L r_{0}^{2} R_{0}}{\left(r_{0}-R_{0}\right)^{2}\left(r_{0}-x\right)}\right\|
$$

as before. And thus in general

$$
\bar{\Psi}_{p} \ll\left\|\frac{n_{.}^{2 p-1} \epsilon^{p} M^{p-1} L r_{0}^{p} R_{0}^{p}}{\left(r_{0}-R_{0}\right)^{2 p-1}\left(x-R_{0}\right)}\right\|, \quad \bar{\Gamma}_{p} \ll\left\|\frac{n^{2 p} \epsilon^{p} \cdot M^{p} L r_{0}^{p+1} R_{0}^{p}}{\left(r_{0}-R_{0}\right)^{2 p}\left(r_{0}-x\right)}\right\|,
$$

as may be proved by induction. If $(17)$ holds, we shall have convergent series for $\gamma_{i j}$ and $\psi_{i j}$ if $r_{0}>|x|>R_{0}$.

Furthermore, from the equations (10) we infer that

$$
\Gamma=P\left(\Theta^{-1} \cdot \Psi\right),
$$

\footnotetext{
* The compound matrix has $\boldsymbol{n}$ terms in each element; hence we get a multiplier $n$.
} 
so that $\gamma_{i j}$ must be an entire function of $x$. Then likewise we infer that

$$
\Psi=\Gamma x^{-m}+N_{m}(\Theta \cdot \Gamma) .
$$

Thus $\left\|\gamma_{i j}\right\|$ and $\left\|\psi_{i j}\right\|$ satisfy (5).

\section{§2. Existence of an equivalent canonical system.}

We are now in a position to answer the question which was raised at the beginning of $\S 1$.

By the hypothesis concerning (2) there are no finite singularities of the functions $a_{i j}(x)$ of $(1)$ exterior to the circle $|x|=R$. Let $x$ describe a closed path exterior to this circle about $x=\infty$. It is easy to show that there will exist $n$ linearly independent particular solutions

$$
\begin{aligned}
& y_{1}^{(1)}, \quad y_{2}^{(1)}, \cdots, \quad y_{n}^{(1)}, \\
& y_{1}^{(n)}, y_{2}^{(n)}, \cdots, \quad y_{n}^{(n)},
\end{aligned}
$$

which undergo the substitution

$$
\begin{aligned}
& \bar{y}_{1}^{(1)}=\rho_{1} y_{1}^{(1)}, \quad \bar{y}_{2}^{(1)}=\rho_{1} y_{2}^{(1)}, \quad \cdots, \quad \bar{y}_{n}^{(1)}=\rho_{1} y_{n}^{(1)}, \\
& \bar{y}_{1}^{(n)}=\rho_{n} y_{1}^{(n)}, \quad y_{2}^{(n)}=\rho_{n} y_{2}^{(n)}, \quad \cdots, \quad \bar{y}_{n}^{(n)}=\rho_{n} y_{n}^{(n)},
\end{aligned}
$$

unless two or more values of $\rho$ are equal, when degenerate cases may occur.*

We will suppose for the present that there exists a fundamental system of this character (19) for which, of course,

$$
\left|y_{i}^{(j)}\right|=c e^{\int\left[a_{11}(x)+a_{22}(x)+\cdot \cdot+a_{n n}(x)\right] d x} .
$$

Define now functions $\theta_{i j}(x)$ by the $n^{2}$ equations

$$
y_{i}^{(j)}=x^{k_{j}} \theta_{i j}(x), \quad\left(k_{j}=\frac{1}{2 \pi \sqrt{-1}} \log \rho_{i}\right) .
$$

It may be inferred at once that the $\theta_{i j}$ are single valued and analytic for $|x|>R$ and that

$$
\left|\theta_{i j}(x)\right|=c x^{-\left(k_{1}+\ldots+k_{n}\right)} e^{\int\left[a_{11}(x)+a_{22}(x)+\ldots+a_{n n}(x)\right] d x} .
$$

Thus the $\theta_{i j}(x)$ fulfill the restrictions of the Lemma. Hence we can determine a set of functions $\lambda_{i j}(x)$ analytic at $x=\infty$ in such a manner that

$$
\sum_{j=1}^{n} \lambda_{i j}(x) \theta_{j k}(x)=x^{-m} \xi_{i k}(x),
$$

the $\xi_{i k}(x)$ being entire functions of $x$ and $\left|\xi_{i k}(0)\right| \neq 0$.

\footnotetext{
* It is here simply a matter of the canonical form of a linear substitution.
} 
The transformation (4) changes $(1)$ into a new system of equations (i) with a fundamental system of solutions

$$
\bar{y}_{i}^{(j)}=x^{k_{j}-m} \xi_{i j}(x) \quad(i, j=1,2, \cdots, n) .
$$

Therefore we can transform a system (1) into an equivalent system whose solutions have no other singularity in the finite $x$-plane except $x=0$, and this singularity will be of the above simple form.

The new system of equations can be written

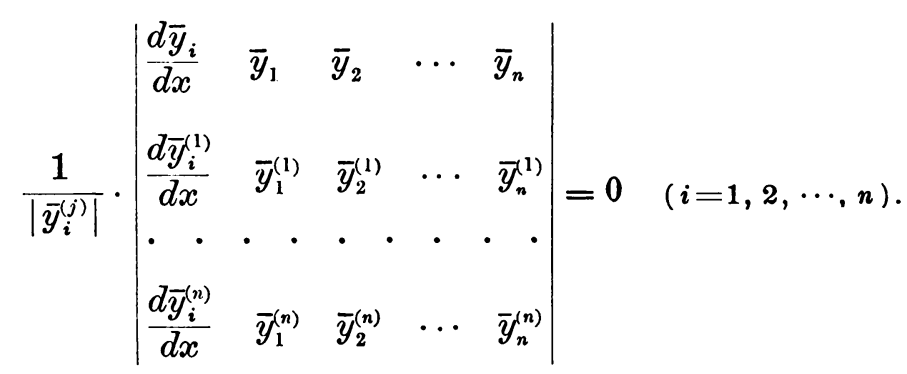

Hence we see that the coefficients $\bar{a}_{i j}(x)$ are quotients of entire functions, and therefore meromorphic in the finite plane with poles only at points for which

$$
\bar{\Delta}=\left|\bar{y}_{i}^{(j)}\right|=0 \text {. }
$$

On the other hand, by a fundamental property of any transformation (4), the new system of equations (20) is of the same form as (1) so that the $\bar{a}_{i i}(x)$ are analytic at $x=\infty$, or have a pole at that point.*

These two properties of the functions $\bar{a}_{i j}$ show that they are rational functions of $x$. Thus the new system of equations equivalent to (1) at $x=\infty$ can be written

$$
\frac{d y_{i}}{d x}=\sum_{j=1}^{n} R_{i j}(x) y_{j},
$$

in which the coefficients $R_{i j}(x)$ are rational. Furthermore it possesses a fundamental system of solutions

$$
y_{i}^{(j)}=x^{k_{i}-m} \xi_{i j}(x),
$$

where the $\xi_{i j}(x)$ are entire functions of $x$ and $\left|\xi_{i j}(0)\right| \neq 0$, and the poles of $R_{i j}(x)$ lie at points for which

$$
\Delta=\left|y_{i}^{(j)}\right|=0 .
$$

* Write the transformation inverse to $(4)$ in the form

$$
y_{i}=\sum_{j=1}^{n} \bar{\lambda} \bar{y}_{j}
$$

in which, of course, the $\bar{\lambda}_{i j}(x)$ are analytic at $x=\infty$, or have a pole at that point. $\Lambda$ direct computation shows that we have

$$
\bar{a}_{l j}=\sum_{k, l=1}^{n} \lambda_{i k} a_{k l} \bar{\lambda}_{l j}+\sum_{k=1}^{n} \bar{\lambda}_{k j} \frac{d}{d x} \lambda_{i k}
$$


By a succession of transformations (4) we shall now remove all the singular points of (21), not at $x=0$ and $x=\infty$. These can only be apparently singular points because of the character of the fundamental system of solutions (22).

Since from (21) we have

$$
\Delta=c e^{\int\left[R_{11}(x)+R_{22}(x)+\ldots+R_{n n}(x)\right] d x}
$$

it is clear that the entire function $\Delta$ is the product of a factor which never vanishes, and of factors of the form $\left(x-x^{\prime}\right)^{\lambda}, \lambda$ being a positive integer. The singular points will lie at $x=x^{\prime}$. These last factors can be removed as follows. Choose constants $c_{1}, c_{2}, \cdots, c_{n}$, not all zero, so that for $x=x^{\prime}$

$$
c_{1} y_{1}^{(j)}+c_{2} y_{2}^{(j)}+\cdots+c_{n} y_{n}^{(j)}=0 \quad(j=1,2, \cdots, n)
$$

This is possible because by hypothesis $\Delta=0$ for $x=x^{\prime}$. Let $c_{k}$ be one of the quantities $c_{i}$ which is not zero. Make the further transformation of the form (4)

$$
\begin{aligned}
& \bar{y}_{i}=y_{i} \\
& \bar{y}_{k}=\frac{c_{1} y_{1}+c_{2} y_{2}+\cdots+c_{n} y_{n} .}{x-x^{\prime}} .
\end{aligned}
$$

The fundamental system of solutions $y_{i}^{(j)}$ of (21) is transformed into another such system of the same character,

$$
\begin{aligned}
\bar{y}_{i}^{(j)} & =y_{i}^{(j)} \\
\bar{y}_{k}^{(j)} & =\frac{c_{1} y_{1}^{(j)}+c_{2} y_{2}^{(j)}+\cdots+c_{n} y_{n}^{(j)}}{x-x^{\prime}} .
\end{aligned}
$$

Accordingly the new system of equations has again the form (21). But we have

$$
\bar{\Delta}=c_{k} \frac{\Delta}{x-x^{\prime}},
$$

so that a factor $x-x^{\prime}$ has been removed from $\Delta$.

In this way one can remove in succession each factor $x-x^{\prime}$, and finally reach the case when $\Delta$ does not vanish except perhaps for $x=0$. Thus a system of equations (21) exists whose solutions have the additional property

$$
\left|y_{i}^{(j)}\right| \neq 0
$$$$
(x \neq 0) .
$$

It follows that the coefficients $R_{i j}(x)$ cannot have any poles except at $x=0$ and $x=\infty$, and so are either polynomials or polynomials multiplied by a positive integral power of $1 / x$. The index of this power does not exceed 1. For suppose we have

$$
R_{i j}(x)=\left(\frac{1}{x}\right)^{\tau}\left\{t_{i j}+t_{i j}^{(1)} x+\cdots\right\} \quad(i, j=1,2, \cdots, n),
$$


in which $\tau>1$ and not every $t_{i j}$ is zero. Substituting $y_{i}^{(j)}=x^{l_{j-}-} \xi_{i j}(x)$ in (21) and equating coefficients of lowest powers of $x$, we obtain

$$
0=\sum_{k=1}^{n} t_{i j} \xi_{k j}(0)
$$

By taking $i=1,2, \ldots, n$ successively it is inferred that $t_{i j}=0(i, j=1,2, \ldots, n)$ since $\left|\xi_{i j}(0)\right| \neq 0$. This is contrary to hypothesis. Therefore we must have

$$
R_{i j}(x)=\frac{P_{i j}(x)}{x},
$$

where $P_{i j}(x)$ denotes some polynomial in $x$.

TheOREM. There always exists a canonical system of equations equivalent to (1) at $x=\infty$,

$$
x \frac{d y_{i}}{d x}=\sum P_{i j}(x) y_{j} \quad(i=1,2, \cdots, n),
$$

in which the $P_{i j}(x)$ are polynomials.

The proof of this theorem has been based on the assumption that there exist $n$ linearly independent solutions of (1) of the character (19). The extension of the above proof to the degenerate cases is illustrated by taking a degenerate case for which $\rho_{n-1}=\rho_{n}$. Then there exist $n$ linearly independent solutions such that we have in place of (19)

$$
\begin{aligned}
& \bar{y}_{1}^{(1)}=\rho_{1} y_{1}^{(1)}, \quad \bar{y}_{2}^{(1)}=\rho_{1} y_{2}^{(1)}, \quad \ldots, \quad \bar{y}_{n}^{(1)}=\rho_{1} y_{n}^{(1)}, \\
& \bar{y}_{1}^{(n-1)}=\rho_{n-1} y_{1}^{(n-1)}, \quad \bar{y}_{2}^{(n-1)}=\rho_{n-1} y_{2}^{(n-1)}, \quad \ldots, \quad \bar{y}_{n}^{(n-1)}=\rho_{n-1} y_{n}^{(n-1)}, \\
& \bar{y}_{1}^{(n)}=\rho_{n-1} y_{1}^{(n)}+y_{1}^{(n-1)}, \quad \bar{y}_{2}^{(n)}=\rho_{n-1} y_{2}^{(n)}+y_{2}^{(n-1)}, \quad \cdots, \quad \bar{y}_{n}^{(n)}=\rho_{n-1} y_{n}^{(n)}+y_{n}^{(n-1)} .
\end{aligned}
$$

In this event we may write

$$
\begin{aligned}
y_{i}^{(j)} & =x^{k_{j}} \theta_{i j}(x), \quad k_{j}=\frac{1}{2 \pi \sqrt{-1}} \log \rho_{j} \quad(i=1,2, \cdots, n ; j=1,2, \cdots, n-1), \\
y_{i}^{(n)} & =x^{k_{n-1}}\left[\theta_{i n}(x)+\frac{1}{2 \pi \sqrt{-1} \rho_{n-1}} \theta_{i, n-1}(x) \log x\right] \quad(i=1,2, \cdots, n) .
\end{aligned}
$$

and the functions $\theta_{i j}(x)$ thus defined will be single-valued and analytic for $|x|>R$.

Furthermore we have

$$
\left|\theta_{i j}(x)\right|=c e^{-\left(k_{1}+\ldots+2 k_{n-1}\right) \int\left[a_{11}(x)+a_{22}(x)+\ldots+a_{n n}(x)\right] d x} .
$$

Thus the functions $\theta_{i j}(x)$ satisfy the restrictions of the lemma of $\S 1$, and a set of multipliers $\lambda_{i j}(x)$ will be determined. If now the transformation (4) be 
made, the new system of equations in $\bar{y}_{i}$ will have a fundamental system of solutions

$$
\begin{aligned}
& \bar{y}_{i}^{(j)}=x^{k_{j}-m} \xi_{i j}(x) \quad(i=1,2, \cdots, n ; j=1,2, \cdots, n-1), \\
& \bar{y}_{i}^{(n)}=x^{k_{n-1}-m}\left[\xi_{i n}(x)+\frac{1}{2 \pi \rho_{n-1} \sqrt{-1}} \xi_{i, n-1}(x)\right] \quad(i=1,2, \cdots, n),
\end{aligned}
$$

in which $\xi_{i j}(x)$ is an entire function and $\left|\xi_{i j}(0)\right| \neq 0$. The rest of the argument in the degenerate case is very like that employed before.

\section{§3. Formal solutions and the polynomials $P_{i j}(x)$.}

It can be shown easily by means of the formal solutions of $(1)^{*}$ that the polynomials $P_{i j}(x)$ cannot all be of degree less than $q+1$.

Let us first consider these formal solutions in the case $q \geqq 0$. If we substitute in (1) formal expressions

$$
\begin{gathered}
y_{i}=e^{P(x)} x^{\kappa} A_{i}(x) \\
P(x)=\alpha \frac{x^{q+1}}{q+1}+\beta \frac{x^{q}}{q}+\cdots+\lambda x, \\
A_{i}(x)=A_{i}+A_{i}^{(1)} \frac{1}{x}+\cdots,
\end{gathered}
$$

and attempt to determine $\alpha, \beta, \ldots, \lambda, \kappa, A_{i}, A_{i}^{(1)}, \cdots$ so that the equations resulting are formally satisfied, and not all the $n$ quantities $A_{i}$ are zero, we shall find that $\alpha$ is one of the $n$ roots of the characteristic equation (3) and that we can then determine

$$
A_{i}, \beta, A_{i}^{(1)}, \cdots, \kappa, A_{i}^{(q+2)},
$$

in succession; thus there arise precisely $n$ formal solutions of (1)

where

$$
y_{1}=S_{1}^{(j)}, y_{2}=S_{2}^{(j)}, \cdots, y_{n}=S_{n}^{(j)} \quad(j=1,2, \cdots, n),
$$

$$
\begin{gathered}
S_{i}^{(j)}=e^{P_{j}(x)} x^{\kappa_{j}} A_{i j}(x) \quad(i, j=1,2, \cdots, n), \\
P_{j}(x)=\alpha_{j} \frac{x^{q+1}}{q+1}+\beta_{j} \frac{x^{q}}{q}+\cdots+\lambda_{j} x, \\
A_{i j}(x)=A_{i j}+A_{i j}^{(1)} \frac{1}{x}+\cdots
\end{gathered}
$$

The first set of equations determining $A_{i j}$ are

$$
A_{i j} \alpha_{j}=\sum_{k=1}^{n} a_{i k} A_{k j} \quad(i, j=1,2, \cdots, n) .
$$

* Schlesinger, Vorlesungen über lineare Differentialgleichungen, pp. 176-182. For a discussion of regular singular points see pp. 122-171. 
It is necessary for us to know also that the formal determinant

$$
\left|S_{i}^{(j)}\right|=e^{P_{i}(x)+\cdots+P_{n}(x)} x^{\kappa_{1}+\cdots+\kappa_{n}}\left\{D+D^{(1)} \frac{1}{x}+\cdots\right\}
$$

does not vanish identically. As a matter of fact, the constant $D=\left|A_{i j}\right|$ is not zero. Otherwise we could find multipliers $m_{1}, m_{2}, \ldots, m_{n}$, not all zero, such that

$$
\sum_{j=1}^{n} A_{i j} m_{j}=0 \quad(i=1,2, \cdots, n) .
$$

Multiplying equations (27) by $m_{j}(j=1,2, \ldots, n)$ and adding, we obtain

$$
\sum_{j=1}^{n} A_{i j} \alpha_{j} m_{j}=0 \quad(i=1,2, \cdots, n) .
$$

A second set of multipliers is $m_{j}^{\prime}=\alpha_{j} m_{j}$; likewise a third set is $m_{j}^{\prime \prime}=\alpha_{j}^{2} m_{j}$. Thus we obtain $n$ sets of multipliers $m_{j}, \alpha_{j} m_{j}, \ldots, \alpha_{j}^{n-1} m_{j}$. It is clear then that we have

$$
\sum_{j=1}^{n} A_{i j} m_{j}\left[c_{0}+c_{1} \alpha_{j}+\cdots+c_{n-1} \alpha_{j}^{n-1}\right]=0
$$

for all values of $c_{0}, c_{1}, \cdots, c_{n-1}$. Since $\alpha_{1}, \alpha_{2}, \ldots, \alpha_{n}$ are distinct we conclude that

$$
A_{i j} m_{j}=0 \text {. }
$$

Hence for any value of $j$ for which $m_{j} \neq 0$ we have

which is impossible.

$$
A_{i j}=0 \quad(i=1,2, \cdots, n),
$$

Let us turn now to consider the formal solutions of any system $(\overline{1})$, equivalent to $(1)$ at $x=\infty$ by means of a transformation (4). The formal solutions of $(\overline{1})$ are defined by the equations

$$
\bar{S}_{i}^{(j)}=\sum_{k=1}^{n} \lambda_{i k} S_{k}^{(j)} \quad(i, j=1,2, \cdots, n) .
$$

Hence $\bar{S}_{i}^{(j)}$ contains the same exponential factor as $S_{i}^{(j)}$, although $\bar{\kappa}_{j}$ may differ from $\kappa_{j}$ by an integer. The formal determinant $\left|\bar{S}_{i}^{(j)}\right|$ is also not identically zero, since we have

$$
\left|\bar{S}_{i}^{(j)}\right|=\left|\lambda_{i j}(x)\right| \cdot\left|S_{i}^{(j)}\right| \cdot
$$

The same results are obtained for the case $q=-1$ except that the exponential multipliers $e^{P_{j}(x)}$ are lacking in the formal expressions $S_{i}^{(j)}$ and $\bar{S}_{i}^{(j)}$.

We will now prove for the case $q \geqq 0$ that the rank of any system of equations ( $\overline{1}$ ) equivalent to (1) at $x=\infty$ is at least $q+1$. Otherwise we must 
have equations like (27) ( $a_{i j}^{(l)}$ being replaced by $\bar{a}_{i j}^{(l)}$ ) to determine the constants $\bar{A}_{i j}$ of the formal solutions of $(\overline{1})$, in which however

These equations are

$$
\bar{a}_{i j}=0 \quad(i, j=1,2, \cdots, n) .
$$

$$
\bar{A}_{i j} \alpha_{j}=0
$$$$
(i, j=1,2, \cdots, n),
$$

whence we see that $\bar{A}_{i j}=0(i=1,2, \ldots, n)$ for any $\alpha_{j}$ not zero. This is impossible.

In particular, then, the rank of the canonical system (25) equivalent to (1) at $x=\infty$ is at least $q+1$. The polynomials $P_{i j}(x)$ cannot all be of degree less than $q+1$.

We shall now prove that the degree of the polynomials $P_{i j}(x)$ can be taken not to exceed $q+1$.

Let us denote the $n$ formal solutions of $(25)$ by $T_{1}^{(j)}, T_{2}^{(j)}, \ldots, T_{n}^{(j)}$ $(j=1,2, \cdots, n)$ where

$$
\begin{aligned}
T_{i}^{(j)}= & e^{P_{j}(x)} x^{l_{j}} B_{i j}(x), \\
& B_{i j}(x)=B_{i j}+B_{i j}^{(1)} \frac{1}{x}+\cdots,
\end{aligned}
$$

$\kappa_{j}-l_{j}$ being an integer. Let $t_{i}$ be the index of the highest power of $1 / x$ which all the $B_{i j}(x)(j=1,2, \ldots, n)$ contain as a factor for fixed $i$. Then we may write

$$
\begin{aligned}
T_{i}^{(j)}=e^{P_{j}(x)} x^{l_{j}+t_{i}} C_{i j}(x), & \\
& C_{i j}(x)=C_{i j}+C_{i j}^{(1)} \frac{1}{x}+\cdots
\end{aligned}
$$

By a succession of transformations (4) of such a character as not to change the form of (25), we now proceed, if necessary, so to transform (25) that

$$
\left|C_{i j}\right| \neq 0 \text {. }
$$

Assume the indices 1 to $n$ so chosen that

$$
t_{1} \leqq t_{2} \leqq \cdots \leqq t_{n} .
$$

If $\left|C_{i j}\right|=0$, one can choose constants $c_{1}, c_{2}, \cdots, c_{n}$ not all zero, such that

$$
c_{1} C_{1 j}+c_{2} C_{2 j}+\cdots c_{n} C_{n j}=0 \quad(j=1,2, \cdots, n) .
$$

Let $c$, be the last one of this set which is not zero. The transformation of the form (4),

$$
\begin{aligned}
& \bar{y}_{i}=y_{i} \\
& \bar{y}_{\triangleleft}=c_{1} x^{t_{\mathrm{t}}-t_{1}} y_{1}+c_{2} x^{t_{\mathrm{o}}-t_{2}} y_{2}+\cdots+c_{\triangleleft} y_{\mathrm{\iota}}, \quad(i \neq s),
\end{aligned}
$$


leaves (25) unaltered in form. The new formal expressions $\bar{T}_{i}^{(j)}$ are the same as $T_{i}^{(j)}$ for $i \neq s$. Also

$$
\begin{aligned}
\bar{T}_{s}^{(j)} & =c_{1} x^{t_{s}-t_{j}} T_{1}^{(j)}+c_{2} x^{t_{s}-t_{i}} T_{2}^{(j)}+\cdots+c_{s} T_{s}^{(j)} \\
& =e^{P_{j}(x)} x^{l_{j}+t_{s}}\left\{\sum_{l=0}^{s} c_{l} C_{l j}+\sum_{l=0}^{s} c_{l} C_{l j}^{(1)} \frac{1}{x}+\cdots\right\} \quad(j=1,2, \cdots, n) .
\end{aligned}
$$

The terms $\sum_{l=0}^{i} c_{l} C_{l j}(j=1,2, \ldots, n)$ are all zero by hypothesis. The formal expressions $\bar{T}_{s}^{(j)}(j=1,2, \ldots, n)$ will contain another factor $1 / x$, and we have increased $t$, by unity or more.

But we have

$$
\left|\bar{T}_{i}^{(j)}\right|=c_{\bullet}\left|T_{i}^{(j)}\right|
$$

directly from the transformation. In this way we can increase $t_{1}, t_{2}, \cdots, t_{n}$ without altering, save by a constant factor, the formal determinant $\left|T_{i}^{(j)}\right|$. Since the determinant contains $1 / x$ at least to the power

$$
l_{1}+l_{2}+\cdots+l_{n}+t_{1}+t_{2}+\cdots+t_{n},
$$

we see that this process of transformation must finally come to an end. This occurs when $(29)$ is satisfied.

When (25) has been transformed so that also (29) holds we may prove at once that the degree $d_{i j}$ of $P_{i j}$ can not exceed $q+1+t_{i}-t_{j}$. In fact, if some of the integers

$$
d_{i j}-\left(q+1+t_{i}-t_{j}\right) \quad(i, j=1,2, \cdots, n)
$$

are positive, let $\tau$ be the greatest of them. Also denote the coefficients of the term

$$
x^{\tau+q+1+t_{i}-t_{j}}
$$

in $P_{i j}(x)$ by $p_{i j}$. Substitute the formal solutions in (25). When coefficients of highest powers of $x$ are equated to zero one obtains

$$
0=\sum_{j=1}^{n} p_{i j} C_{j k} \quad(i, k=1,2, \cdots, n),
$$

which is not possible in view of (29).

We note at this point that the degree of $P_{i j}(x)$ does not exceed $q+1$ if $i \leqq j$. We will now make further transformations so that this will also be true when $i>j$.

Let us first attempt to remove the constant terms of $P_{n j}(x)(j=1,2, \cdots, n-1)$ by a transformation

$$
\bar{y}_{i}=y_{i} \quad(i \neq n), \quad \bar{y}_{n}=c_{n n} y_{n}+c_{n, n-1} y_{n-1}+\cdots+c_{n 1} y_{1},
$$


where $c_{n n}=1$. We will then obtain

$$
\begin{array}{lrl}
\bar{P}_{i j}(x) & =P_{i j}(x)-c_{n j} P_{i n}(x) & (i, j \neq n) \\
\bar{P}_{i n}(x)=P_{i n}(x) & (i \neq . n) \\
\bar{P}_{n k}(x)=\sum_{j=1}^{n} c_{n j} P_{j k}(x)-\sum_{j=1}^{n} c_{n j} P_{j n}(x) c_{n k} & (k \neq n), \\
\bar{P}_{n n}(x)=\sum_{j=1}^{n} c_{n j} P_{j n}(x) . &
\end{array}
$$

It is to be noted that the degree of $\bar{P}_{i j}(x)(i, j=1,2, \cdots, n)$ does not exceed $q+1+t_{i}-t_{j}$. If $p_{i j}^{(0)}$ denotes the constant term of $P_{i j}(x)$ we have to satisfy the conditions

Or if we write

$$
\sum_{j=1}^{n} c_{n j} p_{j k}^{(0)}-\sum_{j=1}^{n} c_{n j} p_{j n}^{(0)} c_{n k}=0 \quad(k=1,2, \cdots, n-1) .
$$

$$
\sum_{j=1}^{n} c_{n j} p_{j n}^{(0)}=\lambda=\lambda c_{n n}
$$

these conditions may be stated in the form

$$
\sum_{j=1}^{n} c_{n j} p_{j k}^{(0)}-\lambda c_{n k}=0 \quad(k=1,2, \cdots, n) .
$$

This gives the equations for $\lambda$,

$$
\left|p_{i j}^{(0)}-\delta_{i j} \lambda\right|=0 \text {. }
$$

Now each transformation employed since we obtained (21) has not affected the form (22) of the solutions. Hence (25) still possesses a fundamental system of solutions of this form. A direct substitution of these solutions in (25) will show that the roots $\lambda$ are the quantities

$$
k_{1}-m, \cdots, k_{n}-m \text {. }
$$

The roots $\lambda$ are therefore distinct and do not differ by integers in the case in which (1) has $n$ solutions of the form (19) where $\rho_{1}, \rho_{2}, \ldots, \rho_{n}$ are distinct, inasmuch as

$$
k_{j}=\frac{1}{2 \pi \sqrt{-1}} \log \rho_{j} \quad(j=1,2, \cdots, n) .
$$

We confine ourselves to this simplest case.

Choose for $\lambda$ any root $k_{n}-m$, determining a set of values $c_{n j}(j=1,2, \ldots, n)$ in which $c_{n n} \neq 0$. Such a root exists, since otherwise the linear equations

$$
\sum_{j=1}^{n-1} c_{n j} p_{j k}^{(0)}-\lambda c_{n k}=0 \quad(k=1,2, \cdots, n-1)
$$

could be satisfied for $n$ values of $\lambda$, and there are only $n-1$ such values. The corresponding transformation $\left(c_{n n}=1\right)$ will remove the desired terms. Also we have $p_{n n}^{(0)}=k_{n}-m$ in the new system of equations. 
Next we remove the constant terms of $P_{n-1, j}(x)(j=1,2, \ldots, n-2)$ by a transformation

$$
\bar{y}_{i}=y_{i} \quad(i \neq n-1), \quad \bar{y}_{n-1}=y_{n-1}+c_{n-1, n-2} y_{n-2}+\cdots+c_{n-1,1} y_{1} .
$$

This can always be accomplished as before and we shall have reduced the constant term of $p_{n-1, n-1}(x)$ to $k_{n-1}-m$. Continuing thus, we finally remove all the constant terms of $P_{i j}(x)(i<j)$ leaving

$$
p_{11}^{(0)}=k_{1}-m, p_{22}^{(0)}=k_{2}-m, \cdots, p_{n n}^{(0)}=k_{n}-m .
$$

When the constant terms are removed, we apply a transformation

$$
\bar{y}_{i}=y_{i} \quad(i \neq \alpha), \quad \bar{y}_{\alpha}=y_{\alpha}+c x^{\tau} y_{\beta}
$$

to remove any term $p_{a \beta}^{(\tau)} x^{\tau}$ of $P_{a \beta}(x)(\alpha>\beta)$ of degree $\tau$ less than $t_{a}-t_{\beta}$. A direct computation shows that we have

$$
\begin{array}{lr}
\bar{P}_{i j}(x)=P_{i j}(x) & (i \neq \alpha, j \neq \beta), \\
\bar{P}_{i \beta}(x)=P_{i \beta}(x)-c x^{\tau} P_{i a}(x) & (i \neq \alpha), \\
\bar{P}_{a j}(x)=P_{a j}(x)-c x^{\tau} P_{\beta j}(x) & (j \neq \beta), \\
\bar{P}_{a \beta}(x)=P_{a \beta}(x)-c x^{\tau}\left[P_{a a}(x)-P_{\beta \beta}(x)+c x^{\tau} P_{\beta a}(x)\right] . &
\end{array}
$$

It is clear that the degree of $\bar{P}_{i j}(x)(i, j=1,2, \ldots, n)$ is not greater than $q+1+t_{i}-t_{j}$ since $\tau<t_{\alpha}-t_{\beta}$.

Let us take $\tau=1$. All the new terms in $\bar{P}_{i j}(x)(i<j)$ are at least of first degree, since a multiplier $x$ appears throughout in them. Furthermore, since $P_{i a}(x)(i>\alpha)$ and $P_{\beta j}(x)(\beta>j)$ contain no constant terms after the previous transformation, it is clear that there are no new terms of first degree except possibly in

$$
\bar{P}_{i \beta}(x) \quad(i<\alpha), \quad \bar{P}_{\alpha j}(x) \quad(\beta<j), \quad \bar{P}_{\alpha \beta}(x) .
$$

Also the terms of the first degree in $\bar{P}_{a \beta}(x)$ can be made zero by a proper choice of $c$, namely such that

$$
p_{a \beta}^{(1)}-c\left(k_{\alpha}-k_{\beta}\right)=0 .
$$

This shows that we may remove the terms of first degree in $P_{i j}(x)\left(t_{i}-t_{j}>1\right)$, by taking them in proper order, namely with increasing $i$ and decreasing $j$.

In the same way we may continue, taking $\tau=2,3, \ldots$, until all terms of $P_{i j}(x)(i>j)$ of degree less than $t_{i}-t_{j}$ are removed. We may therefore write finally

$$
P_{i j}(x)=x^{t_{i}-t_{j}} Q_{i j}(x)
$$

where $Q_{i j}(x)$ is a polynomial for $i>j$. This holds likewise for $i \leqq j$, since then 
$t_{i}-t_{j} \leqq 0$. Also $Q_{i j}(x)$ will be of degree $q+1$ or less, since $P_{i j}(x)$ is of degree $q+1+t_{i}-t_{j}$ or less.

The transformation

will give us a system of equations

$$
y_{i}=x^{t_{i}} \bar{y}_{i}
$$

$$
(i=1,2, \cdots, n)
$$

in which

$$
x \frac{d \bar{y}_{i}}{d x}=\sum_{j=1}^{n} \bar{P}_{i j}(x) \bar{y}_{j}
$$

$\bar{P}_{i j}(x)=x^{t_{j}-t_{i}} P_{i j}=Q_{i j}(x) \quad(i \neq j), \quad \bar{P}_{i i}(x)=P_{i i}(x)+t_{i}=Q_{i i}(x)+t_{i}$.

Thus there exists a canonical system (25), equivalent to (1) at $x=\infty$, in which the degree of $P_{i j}(x)$ does not exceed $q+1$.

We still have at our disposal a linear transformation with constant coefficients,

$$
Y_{i}=\sum c_{i j} y_{j}
$$

In view of the nature of the roots of the characteristic equation (3) we may simplify $P_{i j}(x)$ by this means so that the equivalent canonical system of equations becomes*

$$
\begin{aligned}
x \frac{d Y_{i}}{d x}=\sum_{j=1}^{n} P_{i j}(x) Y_{j}, \\
P_{i j}(x)=p_{i j}^{(0)}+p_{i j}^{(1)} x+\cdots+p_{i j}^{(q)} x^{q} \\
P_{i i}(x)=p_{i i}^{(0)}+p_{i i}^{(1)} x+\cdots p_{i i}^{(q)} x^{q}+\alpha_{i} x^{q+1} .
\end{aligned}
$$

The result which we have obtained is that there exists a canonical system of equations of the form (32) equivalent to (1) at $x=\infty$. This form will be hereafter assumed.

In the case $q=-1$ the canonical system reduces to

$$
x \frac{d Y_{1}}{d x}=\alpha_{1} Y_{1}, \quad x \frac{d Y_{2}}{d x}=\alpha_{2} Y_{2}, \cdots, x \frac{d Y_{n}}{d x}=\alpha_{n} Y_{n} .
$$

But this system of equations is equivalent to (1). Hence, as stated in the introduction, there exists a fundamental system of solutions of (1)

$$
y_{1}^{j \prime}=\lambda_{1 j}(x) x^{a_{j}}, y_{2}^{(j)}=\lambda_{2 j}(x) x^{a_{j}}, \cdots, y_{n}^{(j)}=\lambda_{n j}(x) x^{a_{j}} \quad(j=1,2, \cdots, n),
$$

where $\lambda_{i j}(x)$ are analytic at $x=\infty$ or have a pole at that point. As there are precisely $n$ formal solutions,

$$
\begin{gathered}
y_{1}=x^{a_{j}} A_{1 j}(x), y_{2}=x^{a_{j}} A_{2 j}(x), \cdots, y_{n}=x^{a} A_{n j}(x), \quad(j=1,2, \cdots, n), \\
A_{i j}(x)=A_{i j}+A_{i j}^{(1)} \frac{1}{x}+\cdots,
\end{gathered}
$$

* For an analogous reduction in the case $q=0$ see SchLESINGKR, loc. cit., pp. 183, 184. 
we infer that $\lambda_{i j}(x) \equiv A_{i j}(x)$ and is therefore analytic at $x=\infty$. This is the fundamental result for a regular singular point and is due to Sauvage.*

\section{§4. Generalized Laplace integrals.}

The problem of the paper now resolves itself into a study of the solutions of (32) for $q \geqq 0$.

Let us consider first the case $q=0$. The equations (32) are then

$$
\begin{aligned}
& x \frac{d Y_{1}}{d x}=\left(p_{11}^{(0)}+\alpha_{1} x\right) Y_{1}+p_{12}^{(0)} Y_{2}+\cdots+p_{1 n}^{(0)} Y_{n}, \\
& x \frac{d Y_{2}}{d x}=p_{21}^{(0)} Y_{1}+\left(p_{22}^{(0)}+\alpha_{2} x\right) Y_{2}+\cdots+p_{2 n}^{(0)} Y_{n}, \\
& \cdot . \cdot \cdot \cdot \cdot \cdot \cdot \cdot \cdot \cdot \cdot \cdot \cdot \cdot \cdot \cdot \cdot \cdot \cdot \\
& x \frac{d Y_{n}}{d x}=p_{n 1}^{(0)} Y_{1}+p_{n 2}^{(0)} Y_{2}+\cdots+\left(p_{n n}^{(0)}+\alpha_{n} x\right) Y_{n} .
\end{aligned}
$$

The formal solutions will have the form $\left(T_{1}^{(j)}, T_{2}^{(j)}, \ldots, T_{n}^{(j)}\right)(j=1,2, \ldots, n)$, where

$$
\begin{aligned}
T_{i}^{(j)}= & e^{\alpha, x} x^{l j} B_{i j}(x), \\
& B_{i j}(x)=B_{i j}+B_{i j}^{(1)} \frac{1}{x}+\cdots .
\end{aligned}
$$

Direct substitution shows that

$$
l_{j}=p_{j j}^{(0)} \quad(j=1,2, \cdots, n)
$$

In this system of linear differential equations write

$$
Y_{i}=\int e^{x z} \eta_{i}(z) d z
$$$$
(i=1,2, \cdots, n) \text {. }
$$

By employing the formula

$$
\int e^{x z} x u(z) d z=\left[e^{x z} u(z)\right]-\int e^{x z} d u(z),
$$

in which the path of integration will be subsequently so chosen that the bracketed term may be omitted, we obtain, by equating the coefficients of $e^{x z}$ in the integrands on both sides of (33),

$$
\begin{aligned}
& -\left(z-\alpha_{1}\right) \frac{d \eta_{1}}{d z}=\left(p_{11}^{(0)}+1\right) \eta_{1}+p_{12}^{(0)} \eta_{2}+\cdots+p_{1 n}^{(0)} \eta_{n}, \\
& -\left(z-\alpha_{2}\right) \frac{d \eta_{2}}{d z}=p_{21}^{(0)} \eta_{1}+\left(p_{22}^{(0)}+1\right) \eta_{2}+\cdots+p_{2 n}^{(0)} \eta_{n} \\
& -\left(z-\alpha_{n}\right) \frac{d \eta_{n}}{d z}=p_{n 1}^{(0)} \eta_{1}+(p)_{n 2}^{(0)} \eta_{2}+\cdots+\left(p_{n n}^{(0)}+1\right) \eta_{n}
\end{aligned}
$$

\footnotetext{
* Annales de l'École Normale, series 3, vol. 3 (1886), p. 392. The analogous result for a single equation of the $n$th order is due to Fuchs.
} 
We shall show how to employ (35), thus formally deduced, to obtain an actual representation (34) of the solutions.

The system (35) has $n+1$ regular singular points at $z=\alpha_{1}, z=\alpha_{2}, \cdots, z=\alpha_{n}$ and $z=\infty$, and no other singular points. The exponents at $x=\alpha_{j}$ are all zero except one which has the value $-p_{j j}^{(0)}-1=-l_{j}-1$. These results show that we may choose a fundamental system of $n$ solutions, of which $n-1$ are regular at $x=\alpha_{j}$ and one has the form

$$
\begin{aligned}
\eta_{1}^{(j)}=\left(z-\alpha_{j}\right)^{-l_{j}-1} \mathfrak{P}_{1}^{(j)}\left(z-\alpha_{j}\right), \quad \eta_{2}^{(j)}=\left(x-\alpha_{j}\right)^{-l_{j}-1} \mathfrak{P}_{2}\left(z-\alpha_{j}\right) . \\
\cdots, \eta_{n}^{(j)}=\left(z-\alpha_{j}\right)^{-l_{j}-1} \mathfrak{P}_{n}\left(z-\alpha_{j}\right)
\end{aligned}
$$

in which $\mathfrak{P}_{i}^{(j)}\left(z-\alpha_{j}\right)$ is regular at $z=\alpha_{j}$. This is true except possibly when $l_{j}$ is an integer. We will refer to this possibility later.

The formula (34) will represent a solution of the given canonical system of equations provided we write

$$
\eta_{1}=\eta_{1}^{(j)}, \quad \eta_{2}=\eta_{2}^{(j)}, \cdots, \eta_{n}=\eta_{n}^{(j)},
$$

and the corresponding contour $c_{j}$ of integration is taken to be a "loop circuit" that is, a circuit extending along a (properly chosen) ray from $z=\infty$ to the vicinity of $z=\alpha_{j}$, and then around the point in a negative sense, returning finally to $z=\infty$ along the ray. The direction of the ray must be so chosen that it does not contain another singular point $z=\alpha_{k}$, and that $R(x z)$ (the real part of $x z$ ) is negative along the ray. When this is done there is no difficulty in proving that each set of formulas

$$
Y_{i}^{(j)}=\int_{c_{j}} e^{x z}\left(z-\alpha_{j}\right)^{-l_{j}-1} \mathfrak{P}_{i}^{(j)}\left(z-\alpha_{j}\right) d z \quad(i=1,2, \cdots, n)
$$

represents a solution of (33), at least in certain sectors of the plane.

In the case in which $-l_{j}-1$ is a positive integer the loop circuit is replaced by a straight line path from $z=0$ to $z=\infty$. When $-l_{j}-1$ is negative or zero, there will be a solution which contains a logarithm. We choose this solution to replace $\eta_{1}^{(j)}, \eta_{2}^{(j)}, \ldots, \eta_{n}^{(j)}$. In all cases we obtain a formula for each singular point.

This elegant method of attack (for a single linear differential equation of the $n$th order with rational coefficients) was given by PoINCARE in a fundamental paper.* The method has been more fully developed by HoRN, $\dagger$ who shows that a direct study of the solutions can be made by these formulas in the complete vicinity of $x=\infty$.

When $q>0$ the above method fails. Porncare has indicated a method of

* American Journal of Mathematios, vol. 7 (1885), pp. 217-232.

† Mathematische Annalen, vol. 50 (1897), pp. 525-556.

Trans. Am. Math. Soc. 30 
quadratures reducing the case $q>0$ to the case $q=0$, but the application of this method seems impracticable. *

It is the aim of this paragraph to show that, at least in the case before us, formulas exist for $q>0$, analogous to those given when $q=0 . \dagger$

First let us consider the case $q=1$. The equations (32) are in this case

$$
\begin{aligned}
& x \frac{d Y_{1}}{d x}=\left(p_{11}^{(0)}+p_{11}^{(1)} x+\alpha_{1} x^{2}\right) Y_{1}+\left(p_{12}^{(0)}+p_{12}^{(1)} x\right) Y_{2}+\cdots+\left(p_{1 n}^{(0)}+p_{1 n}^{(1)} x\right) Y_{n}, \\
& x \frac{d Y_{2}}{d x}=\left(p_{21}^{(0)}+p_{21}^{(1)} x\right) Y_{1}+\left(p_{22}^{(0)}+p_{22}^{(1)} x+\alpha_{2} x^{2}\right) Y_{2}+\cdots+\left(p_{2 n}^{(0)}+p_{2 n}^{(1)} x\right) Y_{n}, \\
& x \frac{d Y_{n}}{d x}=\left(p_{n 1}^{(0)}+p_{n 1}^{(1)} x\right) Y_{1}+\left(p_{n 2}^{(0)}+p_{n 2}^{(1)} x\right) Y_{2}+\cdots+\left(p_{n n}^{(0)}+p_{n n}^{(1)} x+\alpha_{n} x^{2}\right) Y_{n}
\end{aligned}
$$

The formal solutions are $\left(T_{1}^{(j)}, T_{2}^{(j)}, \ldots, T_{n}^{(j)}\right)(j=1,2, \cdots, n)$, where

$$
\begin{aligned}
T_{i}^{(j)}=e^{a_{j} \frac{x^{2}}{2}+\beta_{j} x} & x^{l j} B_{i j}(x), \\
& B_{i j}(x)=B_{i j}+B_{i j}^{(1)} \frac{1}{x}+\cdots
\end{aligned}
$$

If these formal expressions be substituted in (36), we obtain

$$
\beta_{j}=p_{j j}^{(1)}
$$$$
(j=1,2, \cdots, n) \text {. }
$$

We shall take as the generalized form of (34) in this case

$$
Y_{i}=\int e^{x^{2} z}\left[\eta_{i 0}(z)+x \eta_{i 1}(z)\right] d z \quad(i=1,2, \cdots, n) .
$$

Now substitute this expression (38) in (36), integrating by parts according to the formula

$$
\int e^{x^{2 z} z} x^{2} u(z) d z=\left[e^{x^{2 z}} u(z)\right]-\int e^{x z z} d u(z),
$$

in order to reduce the terms which contain $x^{3}$ and $x^{2}$ to terms in $x^{1}$ and $x^{0}$ respectively. If the bracketed terms are omitted and the coefficients of $e^{x^{2 z}}$ and $x e^{x^{2 z}}$ in the integrands are then equated, there result the equations :

$$
\begin{aligned}
& -\left(2 z-\alpha_{i}\right) \frac{d \eta_{i 1}}{d z}=\eta_{i 1}+\sum_{j=1}^{n}\left[p_{i j}^{(0)} \eta_{j 1}+p_{i j}^{(1)} \eta_{j 0}\right] \quad(i=1,2, \cdots, n), \\
& -\left(2 z-\alpha_{i}\right) \frac{d \eta_{i 0}}{d z}+\sum_{j=1}^{n} p_{i j}^{(1)} \frac{d \eta_{i 1}}{d z}=2 \eta_{i 0}+\sum_{j=1}^{n} p_{i j}^{(0)} \eta_{j 0} \quad(i=1,2, \cdots, n) .
\end{aligned}
$$

* Horn, Acta Mathematica, vol. 23 (1900), pp. 177-201.

†Cf. Cunningham, Proceedings of the London Mathematical Society, ser. 2, vol. 4 (1906), pp. 374-383. 
Thus we reach a system of $2 n$ linear equations of the first order in $z$ to determine the $2 n$ functions

$$
\eta_{11}, \eta_{21}, \cdots, \eta_{n 1}, \eta_{10}, \eta_{20}, \cdots, \eta_{n 0} .
$$

This system of equations is analogous to (35). It has clearly singular points at $z=\alpha_{1} / 2, z=\alpha_{2} / 2, \cdots, z=\alpha_{n} / 2$ and $z=\infty$, the last of which is a regular singular point. The finite singular points are not in general regular, although a slight consideration proves them to be of rank $q=0$ at most.

It is possible however to make a given one of these singular points $z=\alpha_{r} / 2$ a regular point by first making in (36) the simple preliminary transformation

$$
Y_{i}=e^{-\beta_{r} x} Y_{i} .
$$

The new system of equations $(\overline{36})$, and hence also $(\overline{39})$ will be the same as before except that $p_{i i}^{(1)}$ is replaced by $p_{i i}^{(1)}-\beta_{r}(i=1,2, \ldots, n)$. These $n$ systems of equations (36), in general distinct and obtained by taking $r=1,2, \ldots, n$, replace the single system (35) of the case $q=0$.

The rth system of equations $(\overline{39})$ has a regular singular point at $z=\alpha_{r} / 2$. For the system (39) may be written

$$
\begin{array}{lr}
\frac{d \eta_{i 1}}{d z}=\frac{-1}{2 z-\alpha_{i}}\left\{\eta_{i 1}+\sum_{j=1}^{n}\left[p_{i j}^{(0)} \eta_{j 1}+p_{i j}^{(1)} \eta_{j 0}\right]\right\} & (i=1,2, \cdots, n), \\
\frac{d \eta_{i 0}}{d z}=\frac{-1}{2 z-\alpha_{i}}\left\{2 \eta_{i 0}+\sum_{j=1}^{n} p_{i j}^{(0)} \eta_{j 0}+\sum_{j=1}^{n} \frac{p_{i j}^{(1)}}{2 z-\alpha_{j}}\left[\eta_{j 1}+\sum_{k=1}^{n}\left(p_{j k}^{(0)} \eta_{k 1}+p_{j k}^{(1)} \eta_{k 0}\right)\right]\right\} & (i=1,2, \cdots, n) .
\end{array}
$$

A sufficient condition that $z=\alpha_{r} / 2$ be regular is that there is no pole at $z=\alpha_{r} / 2$ of the second or higher order in the coefficients of $\eta_{11}, \ldots, \eta_{n 1}, \eta_{10}, \ldots, \eta_{n 0}$ in the right-hand members. Clearly such a pole cannot exist except in the second $n$ of these equations when $i=r$, and then only if $p_{r, r}^{(1)} \neq 0$. But in (39) $p_{r r}^{(1)}$ is replaced by $p_{r r}^{(1)}-\beta_{r}$, which vanishes by (37). Hence the statement is proved.

The exponents of the regular singular point $z=\alpha_{r} / 2$ can be verified directly to be all 0 except two, which are

$$
\frac{-l_{r}-1}{2}, \frac{-l_{r}-2}{2}
$$

Hence there exists a solution $\eta_{11}^{(r)}, \cdots, \eta_{n 1}^{(r)}, \eta_{10}^{(r)}, \ldots, \eta_{n 0}^{(r)}$ of the form

$$
\eta_{i 0}^{(r)}=\left(z-\frac{\alpha_{r}}{2}\right)^{\frac{-l_{r}-1}{2}} \mathfrak{P}_{i 0}^{(r)}\left(z-\frac{\alpha_{r}}{2}\right), \quad \eta_{i 1}^{(r)}=\left(z-\frac{\alpha_{r}}{2}\right)^{\frac{-l_{r}-1}{2}} \mathfrak{P}_{i 1}^{(r)}\left(z-\frac{\alpha_{r}}{2}\right),
$$

where $\mathfrak{P}_{i 0}^{(r)}\left(z-\alpha_{r} / 2\right)$ and $\mathfrak{P}_{i 1}^{(r)}\left(z-\alpha_{r} / 2\right)$ are analytic at $z=\alpha_{r} / 2$. Each set of formulas 


$$
\begin{array}{r}
Y_{i}^{(j)}=e^{-\beta_{j} x} \int_{c_{j}} e^{x 2 z}\left(z-\frac{\alpha_{j}}{2}\right)^{\frac{-l_{j}-1}{2}}\left[\mathfrak{P}_{i 0}^{(j)}\left(z-\frac{\alpha_{j}}{2}\right)+x \mathfrak{P}_{i 1}^{(j)}\left(z-\frac{\alpha_{j}}{2}\right)\right] d z \\
(i=1,2, \cdots, n),
\end{array}
$$

will then represent a solution provided that $c_{j}$ is a loop circuit to $z=\alpha_{j} / 2$ so chosen that $R\left[x^{2}\left(z-\alpha_{j} / 2\right)\right]$ is negative along the ray.

If $\left(-l_{j}-1\right) / 2$ is an integer, we can obtain solutions corresponding to the other exponent $\left(-l_{j}-2\right) / 2$ which will not be an integer.

The proof of the validity of the above formulas is exactly like that in the case $q=0$.

Let us now consider the general case when we have

$$
\begin{array}{r}
T_{i}^{(j)}=e^{a_{j} \frac{x q^{+1}}{q+1}+\beta_{j} \frac{x q}{q}+\ldots+\lambda_{j} x} x^{l j} B_{i j}(x), \\
B_{i j}(x)=B_{i j}+B_{i j}^{(1)} \frac{1}{x}+\cdots
\end{array}
$$

We write

$$
Y_{i}=\int e^{x^{q+1} z}\left\{\eta_{i 0}(z)+x \eta_{i 1}(z)+\cdots+x^{q} \eta_{i q}(z)\right\} d z,
$$

where the $\eta_{i j}(z)$ are functions to be determined. First we have by substitution in (32)

$$
\begin{array}{r}
\int e^{x^{q+1} z} \sum_{j=0}^{q} x^{j}\left[(q+1) x^{q+1} z+j\right] \eta_{i j}(z) d z=\sum_{j=1}^{n} \int e^{x^{q+1} z}\left(\sum_{k=1}^{q} p_{i j}^{(k)} x^{k}\right)\left(\sum_{l=1}^{q} x^{l} \eta_{j l}(z)\right) d z \\
+\int e^{x^{q+1}} \alpha_{i} x^{q+1} \sum_{l=0}^{q} x^{l} \eta_{i l}(z) d z \quad(i=1,2, \cdots, n) .
\end{array}
$$

The terms involve $x^{0}, x, \cdots, x^{2 q+1}$. We propose to satisfy each of the equations above by deleting in turn all but the terms in

$$
x^{s} \text { and } x^{q+1+s} \quad(s=0,1, \cdots, q)
$$

and then satifying each of $q+1$ equations resulting. One obtains after the omission of a factor $x^{s}$

$$
\begin{aligned}
\int e^{x^{q+1} z} z & {\left[(q+1) x^{q+1} z+j\right] \eta_{i s}(z) d z } \\
= & \sum_{j=1}^{n} \int e^{x^{q+1} z}\left(\sum_{k+l=s} p_{i j}^{(k)} \eta_{j l}(z)+x^{q+1} \sum_{k+l=q+8+1} p_{i j}^{(k)} \eta_{j l}(z)\right) d z+\int e^{x^{q+1}} \alpha_{i} x^{q+1} \eta_{i s}(z) d z .
\end{aligned}
$$

By an integration by parts

$$
\int e^{x^{++1} z} x^{q+1} w(z) d z=\left[e^{x^{q+1} z} w(z)\right]-\int e^{x^{+1} z} d w(z),
$$

in which the bracketed terms are again cancelled by a subsequent choice of limits, we may transform the coefficient $e^{x^{q+1} z}$ in the integrands so that it is inde- 
pendent of $x$. Equating these coefficients we then obtain in a formal manner the system of $n(q+1)$ differential equations for the functions $\eta_{i s}$,

$$
\begin{aligned}
& -\left[(q+1) z-\alpha_{i}\right] \frac{d \eta_{i s}}{d z}+\sum_{j=1}^{n} \sum_{k+l=q+s+1} p_{i j}^{(k)} \frac{d}{d z} \eta_{j l}=(q+1-s) \eta_{i s}+\sum_{j=1}^{n} \sum_{k+l=s} p_{i j}^{(k)} \eta_{j l} \\
& (i=1,2, \cdots, n ; s=0,1, \cdots, q) \text {. }
\end{aligned}
$$

These equations written in succession for $s=q, q-1, \ldots, 0$ are

$$
\begin{aligned}
& -\left[(q+1) z-\alpha_{i}\right] \frac{d \eta_{i q}}{d z}=\eta_{i q}+\sum_{j=1}^{n} \sum_{k+l=q} p_{i j}^{(k)} \eta_{j l} \quad(i=1,2, \cdots, n), \\
& -\left[(q+1) z-\alpha_{i}\right] \frac{d \eta_{i, q-1}}{d z}+\sum_{j=1}^{n} p_{i j}^{(q)} \frac{d \eta_{j q}}{d z}=2 \eta_{i q-1}+\sum_{j=1}^{n} \sum_{k+l=q-1} p_{i j}^{(k)} \eta_{j l} \\
& (i=1,2, \cdots, n), \\
& -\left[(q+1) z-\alpha_{i}\right] \frac{d \eta_{i 0}}{d z}+\sum_{j=1}^{n} p_{i j}^{(q)} \frac{d \eta_{j 1}}{d z}+\cdots+\sum_{j=1}^{n} p_{i j}^{(0)} \frac{d \eta_{j, q+1}}{d z} \\
& =(q+1) \eta_{i 0}+\sum_{j=1}^{n} \sum_{k+l=0} p_{i j}^{(k)} \eta_{j l} \quad(i=1,2, \cdots, n) .
\end{aligned}
$$

The singular points of (40) are at once seen to be $z=\alpha_{1} /(q+1), z=\alpha_{2} /(q+1)$, $\ldots, z=\alpha_{n} /(q+1)$ and $z=\infty$, the last of which is clearly a regular singular point while the others are of rank $q$ at most.

As in the case $q=1$ we can, however, make a given one of the points $z=\alpha_{r} /(q+1)$ a regular singular point by making a preliminary transformation

$$
\bar{Y}_{i}=e^{-\beta_{r} \frac{x q}{q}-\gamma_{r} \frac{x q-1}{q-1} \cdots-\lambda_{r} x} Y_{i}
$$

The new system $(\overline{32})$, and hence also $(\overline{40})$, will not be changed except that

will be replaced by

$$
p_{i i}^{(q)}, p_{i i}^{(q-1)}, \cdots, p_{i i}^{(0)} \quad(i=1,2, \cdots, n),
$$

respectively.

$$
p_{i i}^{(q)}-\beta_{r}, p_{i i}^{(q-1)}-\gamma_{r}, \cdots, p_{i i}^{(0)}-\lambda_{r}
$$

These $n$ systems of equations $(\overline{40})(r=1,2, \ldots, n)$ will, as in the cases $q=0$ and $q=1$, lead to Laplace integral formulas.

We shall now prove that the system $(\overline{4} \overline{0})$ has a regular singular point at $z=\alpha_{r} /(q+1)$. To prove this fact we note that, in view of the preliminary transformation assumed to have been made, the system (32) admits a formal solution $T_{1}^{(r)}, T_{2}^{(r)}, \cdots, T_{n}^{(r)}$, where

$$
T_{i}^{(r)}=e^{a_{r} \frac{x+1}{q+1}} x^{l_{r}} B_{i r}(x) \quad(i=1,2, \cdots, n) .
$$


Now it is easily proved that if the system (32) has a formal solution of this kind, then the adjoint system of equations,

will have a formal solution

$$
\frac{d z_{j}}{d x}=-\sum_{i=1}^{n} z_{i} P_{i j}(x)
$$

$$
z_{j}(x)=e^{-a_{r} \frac{x^{q+1}}{q+1}} x^{-l_{r}}\left\{C_{j}+C_{j}^{(1)} \frac{1}{x}+\cdots\right\} \quad(j=1,2, \cdots, n),
$$

in which not every $C_{j}(j=1,2, \cdots, n)$ is zero. If this formal solution be substituted in the adjoint system, we obtain the following set of linear equations:

$$
\begin{array}{lll}
\alpha_{r} C_{j}=\alpha_{j} C_{j} & (j=1,2, \cdots, n), \\
\alpha_{r} C_{j}^{(1)}=\alpha_{j} C_{j}^{(1)}+\sum_{i=1}^{n} C_{i} p_{i j}^{(q)} & & (j=1,2, \cdots, n), \\
\cdot & \cdot \cdot \cdot \cdot \cdot & \cdot \\
\alpha_{r} C_{j}^{(q)}=a_{j} C_{j}^{(q)}+\sum_{i=1}^{n}\left(C_{i}^{(q-1)} p_{i j}^{(q)}+\cdots+C_{i} p_{i j}^{(1)}\right) & (j=1,2, \cdots, n) .
\end{array}
$$

From the first $n$ of the above equations we infer that

$$
C_{j}=0 \quad(j \neq r), \quad C_{r} \neq 0 .
$$

Multiply now the $n$ equations of the $(k+1)$ th line in $(40)$ by $C_{i}(i=1,2, \ldots, n)$ respectively, the $k$ th set by $C_{i}^{(1)}(i=1,2, \cdots, n)$, and so on. Then add the first $n k$ equations thus obtained. In view of the above relations between the $B_{i}^{(j)}$ one finds

$$
-\left[(q+1) z-\alpha_{r}\right] \sum_{i=1}^{n}\left\{C_{i} \frac{d \eta_{i, q-k}}{d z}+C_{i}^{(1)} \frac{d \eta_{i, q-k+1}}{d z}+\cdots+C_{i}^{(k)} \frac{d \eta_{i q}}{d z}\right\}=L_{k}(\eta),
$$

in which $L_{k}(\eta)$ denotes a certain expression linear in $\eta_{i j}$ with constant coeffcients. Since $C_{i}=0(i \neq r), C_{r} \neq 0$, we may write this in the form

$$
\begin{array}{r}
\frac{d \eta_{r, q-k}}{d z}=-\frac{1}{C_{r}} \sum_{i=1}^{n}\left\{C_{i}^{(1)} \frac{d}{d z} \eta_{i, q-k+1}+\cdots+C_{i}^{(k)} \frac{d}{d z} \eta_{i q}\right\}+\frac{1}{(q+1) z-\alpha_{r}} L_{k}(\eta) \\
(k=0,1,2, \cdots, q) .
\end{array}
$$

We are now able to prove that $z=\alpha_{r} /(q+1)$ is a regular singular point. This will be proved if we show that when we solve (40) for the derivatives of $\eta_{i j}$ in terms of the $\eta_{i j}$ themselves, the coefficients of the expressions obtained have at $z=\alpha_{r} /(q+1)$ poles of the first order at highest. The expressions for $d \eta_{i q} / d z(i=1,2, \cdots, n)$ certainly satisfy this condition, as the first set of equations (40) show. In solving for $d \eta_{i, q-1} / d z(i=1,2, \cdots, n)$ we distinguish the cases $i \neq r$ and $i=r$. Since in the first case $d \eta_{i, q-1} / d z$ appears with a coefficient $-\left[(q+1) z-\alpha_{i}\right]$ not zero at $z=\alpha_{r} /(q+1)$, it is clear that poles of 
higher than the first order do not appear. To solve for $d \eta_{r, q-1} / d z$ we use equation (41) for $k=1$ and deduce the same fact. In this way we solve for $d \eta_{i q} / d z, d \eta_{i, q-1} / d z, \ldots, d \eta_{i 0} / d z$, each time making use of the appropriate equation (41), to prove that no poles of higher than the first order arise. Thus the statement is finally proved.

The $n(q+1)$ exponents at $z=\alpha_{r} /(q+1)$ are all zero excepting $q+1$ of them, which are $\left(-l_{r}-1\right) /(q+1),\left(-l_{r}-2\right) /(q+1), \cdots,\left(-l_{r}-q-1\right) /(q+1)$. I have not found a direct proof of this fact, such as no doubt exists. An outline of an indirect proof is found in the foot-note p. 462. that

We will have then a solution $\eta_{i j}^{(r)}(i=1,2, \ldots, n ; j=0,1, \ldots, q)$ such

$$
\eta_{i j}^{(r)}=\left(z-\frac{\alpha_{r}}{q+1}\right)^{\frac{-l_{r}-1}{q+1} \mathfrak{P}_{i j}^{(r)}}\left(z-\frac{\alpha_{r}}{q+1}\right),
$$

where $\mathfrak{P}_{i j}^{(r)}\left[z-\alpha_{r} /(q+1)\right]$ is a function analytic at $z=\alpha_{r} /(q+1)$. This leads at once to the formulas

$$
Y_{i}^{(j)}=e^{\beta_{j} \frac{x q+1}{q}+\cdots+\lambda_{j} x} \int_{c_{j}} e^{x q+1 z}\left(z-\frac{\alpha_{j}}{q+1}\right)^{\frac{-l_{j}-1}{q+1}} \sum_{k=0}^{q} x^{k} \mathfrak{P}_{i k}^{(j)}\left(z-\frac{\alpha_{r}}{q+1}\right) d z
$$

where $c_{j}$ is a loop circuit about $z=\alpha_{j} /(q+1)$ so chosen that $R\left\{x^{q+1}\left[z-\alpha_{j} /(q+1)\right]\right\}$ is negative along the ray. These actually represent solutions, as can be proved just as in the cases $q=0$ and $q=1$.

If $\left(-l_{j}-1\right) /(q+1)$ is an integer, one may use solutions corresponding to the remaining non-zero exponents, none of which are integers.

\section{§5. Asymptotic representation.}

If one makes the substitution

$$
w=x^{q+1}\left(z-\frac{\alpha_{r}}{q+1}\right)
$$

in the formulas $(42)$ for $j=r$, there is obtained the expression

$$
Y_{i}^{(r)}=e^{P_{r}(x)} x^{l_{r}} \int_{c_{r}} e^{w} w^{\frac{-l_{r}-1}{q+1}} \sum_{j=0}^{q} x^{j} \Re_{i j}^{(r)}\left(x^{-q-1} w\right) d w,
$$

where $c_{r}^{\prime}$ is a loop circuit to $w=0$. When one expands $\mathfrak{P}_{i j}\left(x^{-q-1} w\right)$ and evaluates the successive terms by means of the formula

$$
\int_{c_{r}^{\prime}} e^{w} w^{\kappa} d w=\left(e^{-\pi \kappa \sqrt{-1}}-e^{\pi \kappa \sqrt{-1}}\right) \Gamma(\kappa+1),
$$

there results for $Y_{i}^{(j)}$ a sum of terms of the same form as in the expression $T_{i}^{(j)}$ plus terms which approach zero as $x$ becomes infinite.*

* For a more complete consideration of a like point see the paper by HoRN previously referred to, Mathematische Annalen, vol. 50 (1897), pp. 527-531. 
In order to state the result concisely we shall modify slightly the conventional definition of asymptotic representation.

Definirion. We may say that $Y_{i}$ is asymptotically represented by $T_{i}^{(r}$ along the ray $\arg x=\phi$, or in symbols

$$
Y_{i} \sim T_{i}^{(r)}, \quad \arg x=\phi,
$$

iffor some sector including arg $x=\phi$ as an interior ray and for all values of $m$,

$$
Y_{i}=e^{P_{r}(x)} x^{k_{r}}\left\{A_{i r}+\frac{A_{i r}^{(1)}}{x}+\cdots+\frac{A_{i r}^{(m-1)}}{x^{m-1}}+\frac{\epsilon}{x^{m}}\right\},
$$

in which lim $\epsilon=0$ as $x$ becomes infinite.

The result is then that we have

$$
Y_{i}^{(j)} \sim T_{i}^{(j)}
$$

along any ray for which $R\left\{x^{q+1}\left[z-\alpha_{j} /(q+1)\right]\right\}$ is negative. These expressions $T_{i}^{(j)}$ are, of course, identical with the $T_{i}^{(j)}$ which occur in the formal solutions of $(32) .^{*}$

The ray of the loop circuit to $z=\alpha_{j} /(q+1)$ may vary in direction as long as it does not intersect one of the remaining $n-1$ finite singular points $z=\alpha_{l} /(q+1)$. It is a simple matter to give the actual sectors on which in general the above integral formulas hold. Denote by $\tau_{1}, \tau_{2}, \cdots, \tau_{N}$ the $N=n(n-1)(q+1)$ rays in angular order for which

$$
R\left\{\left(\alpha_{j}-\alpha_{k}\right) x^{q+1}\right\}=0
$$

and also write $\tau_{N+1}=\tau_{1}+2 \pi$. We assume these rays to be distinct. Furthermore, the real part of some particular one of the differences $\left(\alpha_{j}-\alpha_{k}\right) x^{q+1}$ changes from positive to negative as we pass from the sector $\left(\tau_{m-1}, \tau_{m}\right)$ to $\left(\tau_{m}, \tau_{m+1}\right)$. Denote the subscripts in this difference by $j_{m}$ and $k_{m}$. The ray of the loop circuit $c_{j}$ has an argument intermediate between a certain successive pair of arguments

$$
\arg \left(\alpha_{j_{m^{\prime}}}-\alpha_{k_{m^{\prime}}}\right) \text { and } \arg \left(\alpha_{j_{m}}-\alpha_{k_{m}}\right)
$$

for which $k_{m^{\prime}}=k_{m}=j$. In this case $R\left\{x^{q+1}\left[z-\alpha_{j} /(q+1)\right]\right\}$ remains negative for

The formula (42)

$$
\tau_{m}<\arg x<\tau_{m^{\prime}}+\frac{\pi}{q+1} .
$$

$$
Y_{1}=Y_{1}^{(j)}, Y_{2}=Y_{2}^{(j)}, \cdots, Y_{n}=Y_{n}^{(j)}
$$

* At this point it is easy to see that the exponents at $z=\alpha_{r} /(q+1)$ bave the values above stated. For by comparison of the Laplace integrals and the formal solutions we get these values as possible exponents, and no others. But in the special case for which $P_{i j}(x)=0, P_{i i}=p_{i i}^{(0)}+\alpha_{i} x^{q+1}$, it may be at once proved that the exponents have precisely these values. Then if the coefficients of the polynomials are changed continuously, the exponents are also so changed and hence retain the form specified. 
represents a set of $q+1$ solutions fixed by assigning the sector in which the ray of the loop circuit is to lie. This formula is valid for any one of the $q+1$ corresponding sectors

$$
\tau_{m}<\arg x<\tau_{m^{\prime}}+\frac{\pi}{q+1} .
$$

It is to be noted that $m$ and $m^{\prime}$ are any pair of successive values of $m$ for which $k_{m}=k_{m^{\prime}}=j$.

If now we pass back to the solutions of (1), we infer that there exist solutions $y_{1}, y_{2}, \cdots, y_{n}$ of (1) such that

within any given sector

$$
y_{i} \sim S_{i}^{(j)} \quad(i=1,2, \cdots, n)
$$

$$
\tau_{m}<\arg x<\tau_{m^{\prime}}+\frac{\pi}{q+1} .
$$

This result has also been derived in certain special cases by HoRN,* who has used the method of successive approximation.

\section{§6. Characterization at $z=\infty$.}

At this point we can readily give a characterization of the nature of the solutions of (32) and thence of the system (1) equivalent to it at $x=\infty$.

Theorem. There exist $N=n(n-1)(q+1)$ fundamental systems of solutions of the canonical system (32),

such that

$$
\begin{aligned}
& Y_{1 m}^{(1)}, \quad Y_{2 m}^{(1)}, \cdots, \quad Y_{n m}^{(1)}, \\
& \text { •. } \cdot \cdot \cdot \cdot \cdot \cdot \quad\left(m=1,2, \cdots, N^{\prime}\right. \text {, } \\
& Y_{1 m}^{(n)}, \quad Y_{2 m}^{(n)}, \cdots, \quad Y_{n m}^{(n)},
\end{aligned}
$$

and such that

$$
Y_{i m}^{(j)} \sim T_{i}^{(j)}, \quad \tau_{m} \leqq \arg x<\tau_{m+1},
$$

$$
\begin{array}{ll}
Y_{i, m+1}^{(j)} & =Y_{i m}^{(j)} \\
Y_{i, m+1}^{\left(j_{m}\right)} & =Y_{i m}^{\left(j_{m}\right)}+A_{m} Y_{i m}^{\left(k_{m}\right)},
\end{array}
$$

provided we write $Y_{i, N+1}^{(j)}=e^{2 \pi l_{j} v \overline{-1}} Y_{i 1}^{(j)}$.

Proof. In order to avoid confusion we will state at the outset that the solutions (42) are not in general the solutions referred to in this theorem. The latter retain their asymptotic form throughout minimum sectors, while the former retain it throughout maximum sectors.

*Crelle's Journal, vol. 133 (1907), pp. 19-67 
An immediate conclusion of the results of the preceding paragraph is that there exists for every ray arg $x=\phi$ a set of solutions $Y_{1}^{(j)}, Y_{2}^{(j)}, \ldots, Y_{n}^{(j)}$ $(j=1,2, \ldots, n)$ such that along this ray

$$
Y_{i}^{(j)} \sim T_{i}^{(j)} .
$$

In virtue of this fact it is possible to separate the $x$-plane into some finite number of closed sectors $\sigma$, in each of which there exists a fundamental system of solutions $Y_{1}^{(j)}, Y_{2}^{(j)}, \cdots, Y_{n}^{(j)}(j=1,2, \cdots, n)$ such that $Y_{i}^{(j)} \sim T_{i}^{(j)}$ for $x$ in $\sigma$. We choose these sectors so that the rays arg $x=\tau_{m}$ are included at most one within each sector $\sigma$.

Throughout a sector $\sigma$ not containing an inner ray $\tau_{i}$ every solution $Y_{1}, Y_{2}, \ldots, Y_{n}$ of (32) has a definite asymptotic form. For the general solution of $(32)$ is

$$
Y_{i}=c_{1} Y_{i}^{(1)}+c_{2} Y_{i}^{(2)}+\cdots+c_{n} Y_{i}^{(n)} \quad(i=1,2, \cdots, n),
$$

which gives the asymptotic relationship

$$
Y_{i} \sim c_{1} T_{i}^{(1)}+c_{2} T_{i}^{(2)}+\cdots+c_{n} T_{i}^{(n)} .
$$

The relative magnitudes of these $n$ terms as $x$ becomes infinite in $\sigma$ clearly depend on the relative magnitude of

$$
R\left(\alpha_{1} x^{q+1}\right), \quad R\left(\alpha_{2} x^{q+1}\right), \quad \cdots, \quad R\left(\alpha_{n} x^{q+1}\right) .
$$

This does not change except at the rays $\arg x=\tau_{1}, \tau_{2}, \cdots, \tau_{N}$. Suppose for a moment that indices 1 to $n$ are so chosen that the quantities (45) appear in order of descending magnitude. If $c_{k}$ is the first of the quantities $c_{1}, c_{2}, \cdots, c_{n}$ not zero, one has clearly for $x$ in $\sigma$

$$
Y_{i} \sim c_{k} T_{i}^{(k)} .
$$

Furthermore, since the successive regions $\sigma$ have a ray in common, every solution $Y_{1}, Y_{2}, \ldots, Y_{n}$ preserves its asymptotic form throughout successive sectors $\sigma$ until one which contains a ray $\tau_{1}, \tau_{2}, \ldots, \tau_{N}$ is reached. We may therefore unite into a single sector $\sigma_{m}$ the region $\sigma$ which contain a given ray $\tau_{m}$, and all those which succeed it in angular order up to the one which includes the ray $\tau_{m+1}$, and affirm that on each of these sectors $\sigma_{m}$ there exists a fundamental system $Y_{1}^{(j)}, Y_{2}^{(j)}, \ldots, Y_{n}^{(j)}(j=1,2, \ldots, n)$, for which $Y_{i}^{(j)} \sim T_{i}^{(j)}$ throughout the sector.

Next let us determine the character of the general solution $Y_{1}, Y_{2}, \ldots, Y_{n}$ in one of these sectors $\sigma_{m}$ which includes within it the ray arg. $x=\tau_{m}$. Here again the relative magnitude of the quantities (45) determines the important term in (44). But $R\left\{\boldsymbol{P}_{j_{m}}(x)\right\}$ and $R\left\{\boldsymbol{P}_{k_{m}}(x)\right\}$ change order of magnitude along $\arg x=\tau_{m}$, and moreover

$$
\begin{array}{ll}
R\left\{\left(\alpha_{j_{m}}-\alpha_{k_{m}}\right) x^{q+1}\right\}>0 & \left(\arg x<\tau_{m}\right), \\
R\left\{\left(\alpha_{j_{m}}-\alpha_{k_{m}}\right) x^{q+1}\right\}<0 & \left(\arg x>\tau_{m}\right) .
\end{array}
$$


If we suppose indices so chosen that the quantities (45) are in order of magnitude at the first boundary ray of $\sigma_{m}$, two successive terms of (45), say the sth and the $(s+1)$ th, will change order of magnitude for $\arg x=\tau_{m}$. We see by (44) that the solution $Y_{1}, Y_{2}, \ldots, Y_{n}$ will preserve its asymptotic form in $\sigma_{m}$ unless

$$
c_{1}=c_{2}=\cdots=c_{s-1}=0, \quad c_{s} \neq 0, c_{s+1} \neq 0 .
$$

In this case the solution is of the asymptotic form

$$
c_{\varepsilon} T_{1}^{(s)}, c_{8} T_{2}^{(s)}, \ldots, c_{8} T_{n}^{(s)}
$$

at the initial ray of the sector, and of the asymptotic form

at the terminal ray.

$$
c_{s+1} T_{1}^{(s+1)}, c_{s+1} T_{2}^{(s+1)}, \ldots, c_{s+1} T_{n}^{(s+1)}
$$

If, however, we have two solutions such that at the initial ray of the sector

$$
\begin{aligned}
Y_{i} & \sim c_{i} T_{i}^{(s)} \\
Y_{i}^{\prime} & \sim c_{d+1}^{\prime} T_{i}^{(s+1)}
\end{aligned}
$$$$
(i=1,2, \cdots, n),
$$

we can always choose a combination $Y_{i}^{\prime \prime}=Y_{i}+A Y_{i}^{\prime}$ so that $\left(Y_{1}^{\prime \prime}, Y_{2}^{\prime \prime}, \ldots, Y_{n}^{\prime \prime}\right)$ preserves its asymptotic form $c_{d} T_{1}^{(s)}, c_{c} T_{2}^{(s)}, \ldots, c_{s} T_{n}^{(s)}$ throughout the sector $\sigma_{m}$. For we have

$$
\begin{aligned}
& Y_{i}=c_{s} Y_{i}^{(s)}+c_{s+1} Y_{i}^{(s+1)}+\cdots+c_{n} Y_{i}^{(s)}, \\
& Y_{i}^{\prime}=c_{s+1}^{\prime} Y_{i}^{s+1}+\cdots+c_{n}^{\prime} Y_{i}^{(n)} .
\end{aligned}
$$

The required value of $A$ is $-c_{s+1} / c_{s+1}^{\prime}$.

Let now $Y_{11}^{(j)}, Y_{21}^{(j)}, \ldots, Y_{n 1}^{(j)}(j=1,2, \ldots, n)$ be any fundamental system of solutions of (32) such that

$$
Y_{i 1}^{(j)} \sim T_{i}^{(j)}
$$

for $x$ on $\sigma_{1}$. All these solutions will preserve their asymptotic form throughout $\sigma_{2}$ except perhaps the solution

$$
Y_{11}^{\left(j_{1}\right)}, Y_{21}^{\left(j_{1}\right)}, \cdots, Y_{n 1}^{\left(j_{1}\right)}
$$

in view of the preceding discussion. However, in this case we can choose a constant $A$, so that the solution

$$
Y_{11}^{\left(j_{1}\right)}+A_{1} Y_{11}^{\left(k_{1}\right)}, Y_{21}^{\left(j_{1}\right)}+A_{1} Y_{21}^{\left(k_{1}\right)}, \ldots, Y_{n 1}^{\left(j_{1}\right)}+A_{1} Y_{n 1}^{\left(k_{1}\right)}
$$

preserves its asymptotic form

$$
T_{1}^{\left(j_{1}\right)}, T_{2}^{\left(j_{1}\right)}, \ldots, T_{n}^{\left(j_{1}\right)}
$$

throughout the sector $\sigma_{2}$. Therefore if we write

$$
\begin{aligned}
& Y_{12}^{(j)}=Y_{11}^{(j)} \\
& Y_{12}^{\left(j_{1}\right)}=Y_{i 1}^{\left(j_{1}\right)}+A_{1} Y_{i 1}^{\left(k_{1}\right)}
\end{aligned}
$$


the new fundamental system of solutions $Y_{12}^{(j)}, Y_{22}^{(j)}, \ldots, Y_{n 2}^{(j)}(j=1,2, \ldots, n)$ preserves its asymptotic form throughout the sector $\sigma_{2}$.

Passing now to the sector $\sigma_{3}$, we obtain a third fundamental system of solutions, and so on. Thus we define $Y_{i 2}^{(j)}, Y_{i 3}^{(j)}, \ldots, Y_{i, N+1}^{(j)}$ successively in terms of $\boldsymbol{Y}_{i 1}^{(j)}$.

These fundamental systems of solutions have therefore the desired properties stated in the theorem except that we need not have

$$
Y_{i, N+1}^{(j)}=e^{2 \pi l j^{V}-1} Y_{i 1}^{(j)} \quad(i, j=1,2, \cdots, n) .
$$

These relations, too, will be satisfied when a proper choice of the initial fundamental system

$$
Y_{11}^{(j)}, Y_{21}^{(j)}, \cdots, Y_{n 1}^{(j)} \quad(j=1,2, \cdots, n)
$$

is made. To prove this fact we show first that the final fundamental system of solutions

$$
Y_{1, N+1}^{(j)}, Y_{2, N+1}^{(j)}, \cdots, Y_{n, N+1}^{(j)} \quad(j=1,2, \cdots, n)
$$

is entirely independent of the choice of the initial system (46).

Let

$$
Z_{1 m}^{(j)}, Z_{2 m}^{(j)}, \cdots, Z_{n m}^{(j)} \quad(j=1,2, \cdots, n ; m=1,2, \cdots, N)
$$

be a second set of solutions of the same kind; the constants which correspond to $A_{m}$ will be denoted by $B_{m}$.

Let $R\left(\alpha_{a} x^{q+1}\right)$ be that one of the quantities (45) which is least in magnitude in $\left(\tau_{1}, \tau_{2}\right)$. There can only be one solution of (32) asymptotic to $T_{1}^{(s)}, T_{2}^{(s)}, \ldots, T_{n}^{(s)}$ on $\left(\tau_{1}, \tau_{2}\right)$, and we must have

$$
Y_{i 1}^{(s)}=Z_{i 1}^{(s)} \quad(i=1,2, \cdots, n)
$$

in this sector. Now each pair of the quantities (45) become equal for $2(q+1)$ values of $\tau$,

$$
\tau=\tau^{\prime}, \tau^{\prime}+\frac{\pi}{q+1}, \tau^{\prime}+\frac{2 \pi}{q+1}, \cdots, \tau^{\prime}+\frac{2 q-1}{q+1} \pi .
$$

It is clear, then, that on the sector

$$
\tau_{1} \leqq \arg x<\tau_{1}+\frac{\pi}{q+1}=\tau_{\mu} \quad\left(\mu=\frac{n(n-1)}{2}\right),
$$

each pair of these quantities (45) become equal once and only once. The quantity $R\left(\alpha_{s} x^{q+1}\right)$ will therefore increase over the remaining quantities (45) as $\arg x$ changes from $\tau_{1}$ to $\tau_{1}+\pi /(q+1)$. It is therefore clear that

$$
\begin{aligned}
Y_{i 1}^{(s)} & =Y_{i 2}^{(s)}=\cdots=Y_{i \mu}^{(s)}, \\
Z_{i 1}^{(s)} & =Z_{i 2}^{(s)}=\cdots=Z_{i \mu}^{(s)} .
\end{aligned}
$$


Hence we find

$$
Y_{i m}^{(s)}=Z_{i m}^{(s)} \quad(i=1,2, \cdots, n)
$$

for all values of $m$ less than $\mu+1$.

Next let $R\left(\alpha_{t} x^{q+1}\right)$ be that one of the quantities (45) which is second in magnitude in $\left(\tau_{1}, \tau_{2}\right)$. We must have in this case

$$
Z_{i m}^{(t)}=Y_{i m}^{(t)}+c Y_{i m}^{(s)}
$$

for $m=1,2, \ldots$, until a value $m=\theta$ is reached for which $R\left(\alpha_{f} x^{q+1}\right)$ increases over $R\left(\alpha_{t} x^{q+1}\right)$. It must be remembered that $R\left(\alpha_{t} x^{q+1}\right)$ increases over all the quantities $(45)$ except $R\left(\alpha_{s} x^{q+1}\right)$ in $\left(\tau_{1}, \tau_{\mu}\right)$. Now in virtue of the relations

$$
\begin{aligned}
Y_{i, \theta+1}^{(t)} & =Y_{i \theta}^{(t)}+A_{\theta} Y_{i \theta}^{(s)}, & Y_{i, \theta+1}^{(s)} & =Y_{i \theta}^{(s)}, \\
Z_{i, \theta+1}^{(t)} & =Z_{i \theta}^{(t)}+B_{\theta} Z_{i \theta}^{(s)}, & Z_{i, \theta+1}^{(s)} & =Z_{i \theta}^{(s)},
\end{aligned}
$$

the preceding equality for $m=\theta$ may be written

$$
Z_{i, \theta+1}^{(t)}-B_{\theta} Z_{i, \theta+1}^{(s)}=Y_{i, \theta+1}^{(t)}-A_{\theta} Y_{i, \theta+1}^{(s)}+c Y_{i, \theta+1}^{(s)} .
$$

Also since $R\left(\alpha_{t} x^{q+1}\right)$ is greater than $R\left(\alpha_{t} x^{q+1}\right)$ in $\left(\tau_{\theta}, \tau_{\theta+1}\right)$ we conclude that We have already shown that and we obtain from $(50)$

$$
-B_{\theta}=-A_{\theta}+c \text {. }
$$

$$
Y_{i, \theta+1}^{(s)}=Z_{i, \theta+1}^{(s)} \text {, }
$$

$$
Y_{i, \theta+1}^{(t)}=Z_{i, \theta+1}^{(t)} \text {. }
$$

Since for $m>\theta$, the quantity $R\left(\alpha_{t} x^{q+1}\right)$ will increase over the remaining quantities (45), we infer that

$$
Y_{i m}^{(t)}=Z_{i m}^{(t)} \quad(i=1,2, \cdots \quad \eta)
$$

for all values of $m$ greater than $\theta$.

Likewise we have

$$
Z_{i 1}^{(u)}=Y_{i 1}^{(u)}+c Y_{i 1}^{(t)}+d Y_{i 1}^{(s)}
$$

where $R\left(\alpha_{u} x^{q+1}\right)$ is the third of the quantities (45) in order of magnitude. The relation

$$
Z_{i m}^{(u)}=Y_{i m}^{(u)}+c Y_{i m}^{(t)}+d Y_{i m}^{(s)}
$$

will hold for successive values of $m$, the constants $c$ and $d$ only changing for a value of $m$ such that one of the three quantities

$$
R\left(\alpha_{\imath} x^{q+1}\right), \quad R\left(\alpha_{t} x^{q+1}\right), \quad R\left(\alpha_{u} x^{q+1}\right),
$$

changes order of magnitude at $\arg x=\tau_{m}$. If the first increases over the second, the constant $d$ may change; when the second increases over the third, the constant $c$ becomes zero; when the first increases over the third, the con- 
stant $c$ becomes zero. From and after this fixed value of $m<\mu+1$, for which the first increases over the third, we have

$$
Y_{i m}^{(u)}=Z_{i m}^{(u)} \quad(i=1,2, \cdots, n) .
$$

In this way we prove that after some fixed value of $m<\mu+1$ we shall always have

$$
Y_{i m}^{(j)}=Z_{i m}^{(j)} \quad(i=1,2, \cdots, n),
$$

whatever be the particular value of $j$. Therefore we have

$$
Y_{i m}^{(j)}=Z_{i m}^{(j)} \quad(i, j=1,2, \cdots, n)
$$

for $m=\mu$, and of course for greater values of $m$, in particular $m=N+1$.

Choose $Y_{i 1}^{(j)}$ to be this fixed set $Y_{i, N+1}^{(j)}$ multiplied by $e^{-2 \pi l_{j} \sqrt{-1}}$, and all the conditions of the theorem will be satisfied. This is a possible choice, since $T_{i}^{(j)}$ after a complete circuit of $x=\infty$ becomes $e^{2 \pi l_{j} \sqrt{-1}} T_{i}^{(j)}$, and therefore we have $Y_{i, N+1}^{(j)} \sim e^{2 \pi l_{j} \sqrt{-1}} T_{i}^{(j)}$ in $\left(\tau_{1}, \tau_{2}\right)$. This completes the demonstration.

The theorem whose demonstration has just been completed leads at once to a like theorem concerning the solutions of $(1)$.

Theorem. There exist $N=n(n-1)(q+1)$ fundamental solutions of $(1)$,

such that

$$
\begin{aligned}
& y_{1 m}^{(1)}, y_{2 m}^{(1)}, \cdots, y_{n m}^{(1)}, \\
& \text { •. . . . . . }(m=1,2, \cdots, N+1) \text {, } \\
& y_{1 m}^{(n)}, y_{2 m}^{(n)}, \ldots, y_{n m}^{(n)}
\end{aligned}
$$

and such that

$$
y_{i m}^{(j)} \sim S_{i}^{(j)}, \quad \tau_{m} \leqq \arg x<\tau_{m+1},
$$

$$
\begin{aligned}
y_{i, m+1}^{(j)} & =y_{i m}^{(j)} \\
y_{i m}^{(j, m)} & =y_{i m}^{\left(j_{m}\right)}+A_{m} y_{i m}^{\left(k_{m}\right)},
\end{aligned}
$$$$
\left(j \neq j_{m}\right),
$$

provided we write $y_{i, N+1}^{(j)}=e^{2 \pi l_{j} \sqrt{-1}} y_{i 1}^{(j)}$.

This is a complete characterization of the solutions of $(1)$ in the vicinity of $x=\infty$, since any system of functions $Y_{i m}^{(j)}$ satisfying these conditions will form the solutions of a system of equations (1). The characteristic constants which determine the nature of the solutions are the $n(q+2)$ exponential constants

$$
\alpha_{j}, \beta_{j}, \cdots, \lambda_{j}, \kappa_{j} \quad(j=1,2, \cdots, n),
$$

and the $n(n-1)(q+1)$ transformation constants $A_{1}, A_{2}, \cdots, A_{N}$. Not all of the constants $A_{m}$ are independent, since instead of the particular $n$ formal solutions used we might have multiplied each of these by an arbitrary constant. The new transformation constant would then be altered to

$$
\bar{A}_{m}=\frac{c_{k_{m}}}{c_{j_{m}}} A_{m}
$$


Two sets of transformation constants thus related are considered to be the same. Any two systems (1) and ( $\overline{1})$ with the same characteristic constants (except that $\kappa_{j}$ and $\bar{\kappa}_{j}$ may differ by integers) may be proved to be equivalent at $x=\infty$, and conversely.

The total number of essential characteristic constants is accordingly

$$
n(q+2)+n(n-1)(q+1)-(n-1)=n^{2}(q+1)+1 .
$$

But we can dispose of $n-1$ further constants of (32) by the transformation

$$
\bar{Y}_{i}=c_{i} Y_{i}
$$$$
(i=1,2, \cdots, n) .
$$

It is clear then that the canonical system of equations (32) also has at most $n^{2}(q+1)+1$ essential constants. If it be admitted that no further reduction of (32) is possible, we infer that the characteristic constants are not connected by any necessary relation.

\section{§ 7. Generalized Riemann problem.}

We now propose the following generalization of the Riemann problem: To construct a system of $n$ linear differential equations of the first order with prescribed singular points

of respective rank

$$
x_{1}, x_{2}, \cdots, x_{m}, x_{m+1}=\infty
$$

$$
q_{1}, q_{2}, \cdots, q_{m+1}
$$

and with a given monodromic group, the characteristic constants being assigned for each singular point.

Consider the system of equations

$$
\frac{d y_{i}}{d x}=\sum_{j=1}^{n}\left(\sum_{k=1}^{m} \sum_{l=1}^{q_{k}+2} \frac{A_{i j k l}}{\left(x-x_{k}\right)^{l}}+\sum_{k=0}^{q_{m+1}} B_{i j k} x^{k}\right) y_{j} \quad(i=1,2, \cdots, n),
$$

with singular points $x_{1}, x_{2}, \cdots, x_{m}, x_{m+1}=\infty$ of prescribed rank $q_{1}, q_{2}, \cdots, q_{m+1}$. In this equation appear

arbitrary constants.

$$
n^{2}\left(\sum_{l=1}^{m+1} q_{l}+2 m+1\right)
$$

In order to fix the problem we consider the fundamental system of solutions $\left(y_{1}^{(1)}, \ldots, y_{n}^{(1)}\right), \ldots,\left(y_{n}^{(1)}, \ldots, y_{n}^{(n)}\right)$ such that $y_{i}^{(j)}$ at some point $x=a$ reduces to $\delta_{i j}$, and take the group of this particular fundamental system of solutions to be assigned.

The monodromic group contains $n^{2} m$ arbitrary constants, $n^{2}$ for each fundamental substitution. Furthermore, the characteristic constants are

$$
\sum_{1}^{m+1}\left[n^{2}\left(q_{l}+1\right)+1\right]
$$


in number; but between these constants and those of the group there are $n(m+1)$ relations, since the $n$ exponents at each of the $m+1$ singular points are determined either by the group or by the characteristic constants.

We must further prescribe at each singular point precisely which solutions are to correspond to the canonical fundamental system of solutions given in the second theorem of $\S 6$. Now the group itself determines the correspondence (since it determines the exponents) except for $n$ multiplicative constants. There are accordingly

$$
(n-1)(m+1)
$$

further conditions. Thus there are in all

$$
n^{2} m+\sum_{l=1}^{m+1}\left[n^{2}\left(q_{l}+1\right)+1\right\rfloor-n(m+1)+(n-1)(m+1)
$$

conditions to be satisfied.

The number of conditions equal the number of constants at our disposal. This is the basis on which we postulate the possibility of the general problem.

The University of Wisconsin. 